\title{
LA FORMACIÓN EN LEGÍSTICA: AÚN UNA ASIGNATURA PENDIENTE*
}

\author{
FERNANDO CENTENERA SÁNCHEZ-SECO \\ Doctor en Derecho. Profesor de Filosofía del Derecho \\ Universidad de Alcalá
}

Fecha de recepción: 30.6.2016

Fecha de aceptación: 8.9.2016

\begin{abstract}
Resumen: La formación jurídica tradicional, todavía presente en numerosos ámbitos, ha venido centrando la atención en la interpretación y aplicación del derecho. Sin embargo, este enfoque olvida que la creación de aquel también forma parte de lo jurídico. Este estudio pretende llamar la atención sobre la importancia que tiene la formación en legística, e incentivar experiencias en esta línea en aquellos ámbitos donde apenas existen. Para ello, se aportan razones que justifican esta formación, se considera dónde y cuándo puede tener lugar, se ofrecen las líneas básicas de un posible programa y se presentan algunas ideas metodológicas para llevar a cabo su implementación.
\end{abstract}

Palabras clave: legística; redacción legislativa; formación; docencia.

Abstract: Traditional legal training, which is still present in many areas, has focused on the interpretation and application of the law. However, this approach neglects the fact that the creation of the law is also part of the legal sphere. This study aims to highlight the importance of training in legistics, and to encourage experiences in this area in fields where they are very rare. To that end, the reasons justifying this training are given, where and when it could take place is considered, the basic lines of a possible programme are outlined and some methodological ideas for its implementation are presented.

Keywords: Legistics; legal drafting; training; teaching. 
SUMARIO. I. INTRODUCCIÓN. II. ¿POR QUÉ FORMAR? III. ¿DÓNDE Y CUÁNDO FORMAR? IV. ¿EN QUÉ FORMAR? V. ¿CÓMO FORMAR? 1. Conocimientos y habilidades. 2. Posibles metodologías. VI. CONCLUSIONES.

\section{INTRODUCCIÓN}

En un trabajo publicado en 1987, Gomes Canotilho señalaba que las universidades forman al alumnado para aplicar el derecho, pero no para hacerlo ${ }^{1}$. Esta preocupación se ha ido renovando años después en diferentes estudios, pero no es exclusiva del contexto portugués ${ }^{2}$. El siglo pasado ofrece manifestaciones procedentes de diferentes ámbitos en la misma línea, anteriores y coetáneas a las del profesor citado $^{3}$. Es cierto que en las últimas décadas se han desarrollado numerosas experiencias, pero no ha sido así en todos los países. En nuestros días las palabras de Canotilho siguen siendo aplicables a numerosos contextos académicos, donde la temática que venimos considerando apenas encuentra reflejo en los programas de estudio. Según constatan varios trabajos ${ }^{4}$, el panorama sobre la formación jurídica que

\footnotetext{
*Desarrollé parte de este trabajo en una estancia en la Facultad de Derecho de la Universidad Nova de Lisboa. Quisiera mostrar mi agradecimiento a la profesora Teresa Pizarro y a los profesores João Caupers y Nuno Piçarra por toda su amabilidad, ayuda y conversaciones. Debo agradecer también las apreciaciones y sugerencias de quienes han realizado los exámenes de este trabajo.

${ }^{1}$ GOMES CANOTILHO, J. J. "Relatório sobre programa, conteúdos e métodos de um curso de teoria da legislação", Boletim da Faculdade de Direito, LXIII, 1987, p. 409.

${ }^{2}$ CAUPERS, $J$. "Relatório sobre o programa, conteúdo e métodos de uma disciplina de Metódica da Legislação", Legislação. Cadernos de Ciencia de Legislação, 35, 2003, p. 5; TAVARES DE ALMEIDA, M. "Legista - uma nova profissão", Legislação. Cadernos de Ciencia de Legislação, 41, 2005, p. 40.

${ }^{3}$ Aunque en diferentes momentos cronológicos (algunos de los cuales nos retrotraen a la primera mitad del siglo pasado) y aun cuando en varios contextos se observan importantes avances en la fecha actual y también mucho antes, la cuestión se diagnostica en FULLER, L. L. "What the Law Schools Can Contribute to the Making of Lawyers", Journal of Legal Education, 1, 2, 1948, pp. 192, 193 (en relación al ámbito estadounidense); DICK, R. C. Legal Drafting in Plain Language, Ontario, 1995, p. 261 (a propósito del contexto canadiense); DUPRAT, J.-P. "The French »learning « legislator: the improvements of an indirect proces", AA.VV. (Mader, L. y Moll, C., ed.) The Learning Legislator, Baden-Baden, 2009, p. 160 (en relación al ámbito francés); o GWIZDZ, A. "Organization of the Post-Graduate Course in Legislation at the University of Warsaw", AA.VV. (Karpen, U. y Delnoy, P., ed.) Contributions to the Methodology of the Creation of Written Law, Baden-Baden, 1996, p. 108 (a propósito del ámbito polaco).

${ }^{4}$ En el ámbito mexicano se acusan carencias en lo que respecta a la formación, como así se constata en MURO RUIZ, E. "Enseñanza de la técnica legislativa", Academia. Revista sobre enseñanza del Derecho, 11,2008 , pp. 63, 64, 90. Otro testimonio interesante en la misma línea, esta vez desde el contexto chileno, lo encontramos en PINEDA GARFIAS, R. "Teoría de la Legislación. Algunos planteamientos generales", Revista de Derechos Fundamentales, 3, 2009, pp. 137, 138, 151, 152. El caso español también puede enmarcarse dentro de esta relación. Aunque en él encontramos recursos interesantes, como así lo certifica la referencia AA.VV. Curso de técnica legislativa GRETEL, Madrid, 1989, desde hace tiempo se viene constatando la ausencia de la técnica legislativa en los estudios de derecho, al tiempo que se reclama una apertura a las ciencias sociales y humanísticas. Sobre ello SALVADOR CODERCH, P. "Elementos para la definición de un programa de técnica legislativa", AA.VV. (Cavero Gómez, M., coord.) La función legislativa de los parlamentos y la técnica de legislar, Madrid, 2000, p. 50. Más recientemente, se ha hecho referencia a la ausencia de formación lingüística en los planes universitarios de derecho en COMISIÓN DE EXPERTOS MODERNIZACIÓN DEL LENGUAJE JURÍDICO. Informe de la Comisión de modernización del lenguaje jurídico, [s. 1.], 2001, p. 16. Disponible en: http://lenguajeadministrativo.com/wp-content/uploads/2013/05/cmlj-recomendaciones.pdf. Fecha de consulta: $24 / 04 / 2016$.
} 
encontramos actualmente en países como México, Chile o España se caracteriza por otorgar un protagonismo, prácticamente exclusivo, a la interpretación y aplicación de las normas, sin apenas abordar la actividad creadora del derecho ${ }^{5}$. Este estudio pretende poner de manifiesto la relevancia de este último ámbito, que consideraremos en sentido estricto, es decir, como el relativo a la producción de disposiciones legislativas, reglamentarias, etc. Aquel alcanza a la redacción de textos legales, pero también a otras vertientes de análisis interesantes que abordaremos en las siguientes páginas. Esta circunstancia ha motivado que utilicemos el término legística, que hace referencia a un contexto más amplio que aquel que comprende el de drafter, palabra procedente de Reino Unido que alude a la redacción legislativa especializada. La legística tiene una dimensión formal, que contempla cuestiones que tienen que ver con la comprensión de las normas, jugando en este ámbito un papel importante la redacción de aquellas. Pero además de ello comprende también una dimensión material, que se ocupa de la determinación del contenido de las normas. En este sentido la atención se centra en aspectos tales como la definición del problema, los objetivos a conseguir, los medios para ello, etc. ${ }^{6}$ Conviene además apuntar que en relación a la primera parcela señalada, en este estudio no consideraremos la redacción de normas como un contexto de racionalidad estanco, sino como una parcela que debe abordarse junto con otros niveles de racionalidad $^{7}$ (en el apartado cuarto de este trabajo nos referiremos de manera más pormenorizada a todos estos aspectos).

A la hora de desarrollar los contenidos en este estudio hemos planteado la siguiente estructura. En primer lugar dedicamos el apartado siguiente a las razones que justifican la formación en legística. A continuación, en los espacios posteriores pretendemos responder a cuestiones que son ineludibles de cara a posibles implementaciones futuras: en qué formar, dónde, cuándo y cómo. Para llevar a cabo buena parte de este desarrollo hemos tenido en cuenta experiencias de numerosos países, donde o bien puede observarse una tradición en relación a la formación que nos proponemos tratar, o bien es posible localizar prácticas interesantes desarrolladas en las décadas recientes. A la vista de este recurso podría objetarse que los sistemas de redacción tienen características diferentes, que los ordenamientos jurídicos poseen particularidades y que ello podría poner en cuestión la propuesta de transponer contenidos de unos contextos a otros. Ciertamente, las diferencias condicionan la matización o el desarrollo particular, en función de las características del contexto jurídico de que se trate. Sin embargo, también debe apuntarse que existen una serie de cuestiones que son aprovechables, aunque su implementación pormenorizada deba

\footnotetext{
${ }^{5}$ Ello, no obstante, no quiere decir que en estos contextos no exista interés por la temática que nos ocupa. Aquel se confirma con el peso de la producción doctrinal.

${ }^{6}$ Sobre ello TAVARES DE ALMEIDA, M. “Legista...”, cit., pp. 38, 39; CAUPERS, J. "Relatório sobre o programa...", cit., p. 11; MORAND, C.-A. "Préface", AA.VV. (Morand, C.-A., dir.) Légistique formelle et matérielle, Aix-en-Provence, 1999, s. p.

${ }^{7}$ El análisis que presentamos entendemos que se acerca a lo que Marcilla Córdoba denomina "técnica legislativa maximalista", que tiene por objeto diseñar "instrumentos o medios para mejorar la legislación, medios que habrían de ser consistentes con los fines que se estiman justificados o legítimos, e idóneos para alcanzarlos". MARCILLA CÓRDOBA, G. Racionalidad legislativa. Crisis de la ley y nueva ciencia de la legislación, Madrid, 2005, p. 290. Sobre la cuestión también MARCILLA CÓRDOBA, G. "Justificación de las decisiones legislativas: un corolario del estado constitucional", AA.VV. (Gascón Abellán, M., coord.) Argumentación jurídica, Valencia, 2014, p. 487. No obstante, a nuestro modo de ver la legística abarca un ámbito de análisis más extenso que el de la expresión citada.

${ }^{8}$ Este es el parecer de XANTHAKI, H. Drafting Legislation. Art and Technology of Rules for Regulation, Oxford, 2014, pp. 362-365 (que considera la transferibilidad en términos de utilidad no solo posible, sino deseable, habida cuenta de la existencia de problemas transnacionales); CARRILLO GARCÍA, C. Y.
} 
venir marcada y detallada por las circunstancias concretas del ámbito en el que se pretendan insertar. Este estudio se centra principalmente en dichas aportaciones. En consecuencia con lo anterior, no tiene vocación de entrar en concreciones, salvo puntualmente y de modo ejemplificativo. Además de considerar las experiencias señaladas, en estas páginas también hemos tenido en cuenta numerosas referencias doctrinales, algunas cuestiones abordadas en nuestra investigación, que a nuestro modo de ver son aprovechables también en este contexto, y determinados aspectos de prácticas desarrolladas en nuestra experiencia docente ${ }^{9}$.

\section{II. ¿POR QUÉ FORMAR?}

Las razones que pueden aportarse para responder a la cuestión que encabeza este epígrafe son varias. En primer lugar, podemos referirnos a las de carácter académico. Como ya adelantamos en la introducción, el programa de buen número de facultades de derecho se desarrolla en base al punto de vista de la interpretación y aplicación del derecho. Tal perspectiva preside el ámbito teórico, pero también el práctico. Por lo general, los casos que se proponen desde este punto de vista sitúan al alumnado en el puesto de quien ha de defender o acusar a alguien en un tribunal, o de quien debe juzgar en el mismo contexto. Probablemente, la máxima expresión práctica de ello sea la celebración de juicios ficticios en las facultades.

Cabría, no obstante, preguntarse si la perspectiva anterior, que parece tan asumida $^{10}$, resulta acorde con lo que realmente supone el fenómeno jurídico en toda su

“Calidad de las leyes. Algunos puntos críticos”, Legislação. Cadernos de Ciencia de Legislação, 52, 2010, p. 27 (que entiende que los problemas sobre calidad legislativa suelen ser los mismos y que frente a ellos se han propuesto las mismas soluciones); KARPEN, U. "Law Drafting and the Legislative Process: outline of a training course for law drafters", AA.VV. (Mader, L. y Moll, C., ed.) The Learning Legislator, Baden-Baden, 2009, p. 11 (que se refiere a los principios de una redacción clara y concisa); MARCELLO, D. A. "Legislative Learning in the United States and Europe: How jurisdictions differ in training drafters and enacting laws", AA.VV. (Mader, L. y Moll, C., ed.) The Learning Legislator, BadenBaden, 2009, pp. 73 y ss. (que aun constatando diferencias entre Estados Unidos y Europa, considera la necesidad de trabajar en todo caso bajo los principios de un lenguaje claro).

${ }^{9}$ Estos últimos, no obstante, en lo que respecta a la asignatura de Redacción de Textos Legales que impartimos en la Universidad de Alcalá, se consideran desde un punto de vista general, como una opción más dentro del conjunto de posibilidades docentes que aquí aparecerán. Un desarrollo algo más pormenorizado puede encontrarse en CENTENERA SÁNCHEZ-SECO, F. "Recursos para formar en redacción de textos legales", Revista de Llengua i Dret, 65, 2016, pp. 136-148.

${ }^{10}$ Entre las razones de esta circunstancia podemos considerar la tradición positivista dominante a lo largo del siglo XX, que se enfrenta al enfoque interdisciplinar de la redacción en textos legales, o la ideología neoliberal partidaria del recurso a instrumentos no coercitivos. Sobre estas cuestiones ZAPATERO, V. El Arte de Legislar, Pamplona, 2009, pp. 38 y ss.; MORAND, C.-A. "Éléments de légistique formelle et matérielle”, AA.VV. (Morand, C.-A., dir.) Légistique formelle et matérielle, Aix-en-Provence, 1999, p. 36; MARCILLA CÓRDOBA, G. "Argumentación legislativa y teoría estándar de la argumentación jurídica”, AA.VV. (Gascón Abellán, M., coord.) Argumentación jurídica, Valencia, 2014, p. 504. Acerca de los aspectos señalados pueden consultarse además las siguientes referencias, en las que se analiza de manera pormenorizada el papel de la ley en el Estado social: MARCILLA CÓRDOBA, G. Racionalidad legislativa..., cit., pp. 155 y ss.; MARCILLA CÓRDOBA, G. "Sobre la necesidad de una nueva ciencia de la legislación", AA.VV. (Carbonell, M., Thalía, S., coord.) Elementos de técnica legislativa, México, 2000, pp. 94 y ss.; GALIANA SAURA, A. La legislación en el Estado de Derecho, Madrid, 2003, pp. 25 
extensión. La cuestión probablemente haya pasado desapercibida, pero es suficiente con una breve reflexión para darnos cuenta del gran olvido; la labor de quienes crean el derecho apenas es visible en el sistema de formación que venimos considerando. Si se quiere ofrecer una percepción completa de lo jurídico es ineludible tener en cuenta este ámbito $^{11}$; y si asentimos a dicho argumento, al tiempo habremos de reconocer que sin la formación que nos ocupa los programas presentan una visión incompleta de lo jurídico. Sin embargo, eso no es todo, no al menos si se tiene en cuenta que las cuestiones que importan al ámbito que nos interesa, de algún modo se proyectan en los campos de la interpretación y aplicación normativa. Una ley que se haya producido de forma deficiente, por ejemplo, muy probablemente originará problemas de interpretación y aplicación $^{12}$. Nos encontramos, por tanto, con que el proceso de creación del derecho tiene lazos de conexión con el de interpretación y aplicación normativa. Desde la perspectiva de la argumentación jurídica podría decirse que si aquella se funda en la premisa de la racionalidad de la ley, esta debería tener reconocida cierta parcela de atención; así al menos habría de ser si se pretende aportar un conocimiento completo del ámbito al que nos referimos ${ }^{13}$. De este modo, deberá entenderse que un buen análisis de la interpretación y aplicación del derecho demanda ineludiblemente también la consideración de aquel que se dedica a la creación de las normas. Si no se apuesta por un programa que refleje esta conjunción, el resultado que obtendremos será, sí, una formación en el primer modelo señalado, pero no precisamente la adecuada ${ }^{14}$ en términos generales.

En base a lo anterior cabría concluir que la formación en legística no solo enriquece el currículum universitario, sino que además lo dota de más calidad y coherencia. En relación a todo ello podemos considerar otras razones que aparecen conectadas con lo ya dicho. Nos referimos ahora a la justificación de la formación que nos ocupa, a propósito de la futura vida profesional del alumnado de derecho. Así, por ejemplo, cuando hablamos de redacción legislativa, la primera imagen que nos viene a la mente es la de quienes dedican su vida profesional a la redacción de leyes. Probablemente este sea el ámbito por antonomasia en el que se pone en práctica la labor en cuestión, pero no es el único. Podemos pensar también en otras profesiones en las que no se redactan propiamente leyes, pero sí otro tipo de normas, como por ejemplo reglamentos o directrices administrativas ${ }^{15}$. Es más, existen fuentes que adoptando una acepción amplia de redacción legislativa, incluyen en este ámbito a quienes se dedican al ejercicio de la abogacía, ya que esta profesión requiere la redacción de un buen número de documentos, tales como contratos, testamentos, etc. ${ }^{16}$ Cabría preguntarse, no

y ss. Sobre otras posibles razones también CAUPERS, J. "Relatório sobre o programa...", cit., pp. 6-9, que se refiere al hecho de considerar el discurso normativo como algo estanco, o a la idea de que hacer leyes no es algo que tenga reglas que se puedan enseñar, sino más bien una cualidad innata.

${ }^{11}$ ZAPATERO, V. "De la jurisprudencia a la legislación", Doxa, 15-16, 1994, p. 769; SALVADOR CODERCH, P. "Elementos para la definición...", cit., p. 50. Mucho tiempo antes, aunque a propósito de un contexto jurídico diferente, la solicitud se encuentra en FULLER, L. L. "What the Law Schools Can Contribute...", cit., p. 192 y 193.

${ }^{12}$ CAUPERS, J. "Relatório sobre o programa...", cit., p. 21.

${ }^{13}$ MARCILLA CÓRDOBA, G. "Argumentación legislativa y teoría estándar...", cit., p. 505.

${ }^{14}$ FULLER, L. L. "What the Law Schools Can Contribute...", cit., pp. 193, 194.

${ }^{15}$ MORAND, C.-A. "Préface", cit., s. p. Pensamos que también se refiere a ello CARRILLO GARCÍA, C. Y. "Calidad de las leyes...", cit., p. 29.

${ }^{16}$ Seguimos en este punto a FULLER, L. L. "What the Law Schools Can Contribute...", cit., p. 193. 
obstante, cómo se pueden llevar a cabo tareas como las descritas si quienes se ocupan de ellas no han recibido formación, o cuáles pueden ser los resultados ante tal panorama. Muy probablemente no serán satisfactorios.

Con razón se ha dicho que la legislación de calidad depende del nivel de la formación que estamos considerando ${ }^{17}$. Ciertamente, las facultades de derecho tienen una responsabilidad histórica en el desarrollo de una materia que se demuestra cada vez más útil ${ }^{18}$. Esta responsabilidad debe asumirse, si realmente se aboga por una legislación de calidad. La reflexión puede ser la misma en relación a los otros colectivos que consideramos anteriormente. Así, por ejemplo, quien termina los estudios de derecho y comienza a trabajar en un despacho, únicamente cuenta con sus recursos para redactar documentos que deben cumplir tanto con lo que se solicita desde el punto de vista jurídico como con los intereses de la clientela a la que atiende. Esta tarea difícilmente se podrá llevar a cabo si no se ha obtenido la formación apropiada. Podría replicarse, no obstante, que, por ejemplo, es posible recurrir a modelos de documentos que se tengan a mano. Sin embargo, por una parte cabría preguntarse si ello es suficiente y por otra, si no estamos más que ante una vía de perpetuación de defectos que han de evitarse ${ }^{19}$.

Como ha acontecido en otros ámbitos durante diferentes etapas ${ }^{20}$, en numerosos contextos actuales resulta evidente que los estudios en derecho no son suficientes para tener éxito en la labor que centra nuestra atención. Conviene apuntar, no obstante, que con ello no se está queriendo decir que la inclusión de una formación en esta línea, dentro de los estudios ordinarios de derecho, sea suficiente para desempeñar cualquiera de las opciones profesionales señaladas. Principalmente en el caso de la redacción de leyes habrá que decir que no. Sin embargo, junto con ello no deberían olvidarse dos ideas. Por una parte, que la formación en los estudios ordinarios sí que puede suponer un comienzo en la carrera de redacción de textos legales que, además, podría incrementarse con otros cauces en el postgrado. Por otra parte, que por lo que respecta a otros ámbitos donde también han de desarrollarse labores de redacción, la formación en los estudios ordinarios probablemente suponga una gran ayuda. En definitiva, de lo que se trata con todo ello es de aportar las herramientas necesarias para formar a profesionales competentes. Per se este objetivo ya es valioso, por cuanto constituye una contribución a una labor profesional de calidad. Sin embargo, esta cuestión tiene también una interesante lectura desde el punto de vista de la deontología, cuya relevancia se presenta a todas luces si se tiene en cuenta, como veremos enseguida, el impacto que tiene la labor que nos ocupa en la consecución de determinados valores.

\footnotetext{
${ }^{17}$ KARPEN, U. "Improving Democratic Development by Better Regulation", AA.VV. (Stefanou, C. y Xanthaki, H., ed.) Drafting Legislation. A Modern Approach, England, 2008, p. 163; MADER, L. "Legistic Training and Education in Switzerland", AA.VV. (Mader, L. y Moll, C., ed.) The Learning Legislator, Baden-Baden, 2009, p. 47; VAN LOCHEM, P. J. P. M. "The Dutch Academy for Legislation”, AA.VV. (Mader, L. y Moll, C., ed.) The Learning Legislator, Baden-Baden, 2009, p. 64; DRINÓCZI, T. "Quality Drafting-The case of Hungary", Legisprudence. International Journal for the Study of Legislation, IV, 2, 2010, p. 168.

${ }^{18}$ MORAND, C.-A. "Préface", cit., s. p.

${ }^{19}$ DICK, R. C. Legal Drafting..., cit., p. 261.

${ }^{20}$ GWIZDZ, A. "Organization of the Post-Graduate Course...", cit., p. 108.
} 
Dentro del ámbito deontológico se ha considerado que uno de los primeros deberes que han de observarse es el de la competencia profesional, y esta únicamente puede conseguirse si, de nuevo, existe una formación consistente ${ }^{21}$. En el programa de materias pueden observarse variaciones, en función del modelo docente que se implemente. Por nuestra parte podemos decir que en base a la experiencia docente que tenemos, la formación alcanza a aspectos de lingüística, a los relativos a otros niveles de racionalidad de la legística y a los de carácter jurídico que sean necesarios para desarrollar las tareas que se pretendan realizar.

Aparte de las razones académicas y profesionales, debemos referirnos también a otras que tienen que ver con lo que supone el Estado de derecho. A fin de cuentas, el hecho de elaborar textos legales de calidad es una apuesta por las cotas más altas posibles de legalidad ${ }^{22}$; aunque eso no es todo, porque la labor en cuestión también cumple una importante función con respecto a los valores del ordenamiento jurídico. En relación a estos últimos debemos referirnos en primer lugar a la seguridad jurídica, con la que se aporta certeza ${ }^{23}$ acerca del contenido de los derechos y obligaciones, pero no exclusivamente. La consecución de la seguridad repercute positivamente en otros valores, como por ejemplo la libertad. Si la ciudadanía no tiene problemas para entender, en base a los textos normativos, cuáles son las conductas que puede llevar a cabo, las practicará sin temor a que en ello encuentre obstáculo o sanciones ${ }^{24}$. Cabría además añadir que la seguridad y la certeza que la misma ofrece suponen un factor importante para facilitar el desarrollo económico. Piénsese, por ejemplo, en la fiabilidad que aporta un contrato con una buena redacción ${ }^{25}$, o en las leyes que regulan el comercio elaboradas en el mismo sentido. Puede decirse que para lograr la justicia no es suficiente con la eficiencia. Ello es cierto, pero principalmente en nuestro tiempo, la

\footnotetext{
${ }^{21}$ ZAPATERO, V. El Arte de Legislar, cit., pp. 288-292.

${ }^{22}$ PRIETO DE PEDRO, J. "Lenguaje jurídico y Estado de Derecho", Revista de Administración Pública, 140, 1996, pp. 116-119.

${ }^{23}$ Sobre la cuestión es interesante el trabajo de MARCILLA CÓRDOBA, G. "Justificación de las decisiones legislativas...", cit., pp. 463 y ss.

${ }^{24}$ Podría señalarse que lo dicho no es garantía de acción libre, pues el ámbito de acción permitido puede ser estrecho. Sobre ello TAMANAHA, B. Z. "A Concise Guide to the rule of law", AA.VV. (Mader, L. y Moll, C., ed.) The Learning Legislator, Baden-Baden, 2009, p. 23. También podría considerarse que la claridad de las normas, per se, no es sinónimo de justicia, pero lo que es indudable es que en el Estado constitucional sí constituye una parte de la justicia, por cuanto aporta certeza acerca de los derechos y obligaciones que tenemos, además de sus límites. Nos hemos ocupado de esta cuestión en CENTENERA SÁNCHEZ-SECO, F. "Buscando el valor de la claridad de las normas: algunas reflexiones desde el pensamiento de Lon L. Fuller", Bajo Palabra. Revista de Filosofía, 10, 2015, p. 65. La misma reflexión podría aplicarse al planteamiento sobre la libertad, pues el Estado constitucional no tiene únicamente en cuenta la concepción restringida de Estado de derecho, sino que además comprende aspectos tales como la democracia y los derechos humanos. A propósito de este punto de vista, quizá sea interesante señalar que la solicitud de calidad normativa viene reforzada por la jurisprudencia constitucional, que solicita reducir incertidumbres en lo que respecta a la regulación que afecte a derechos fundamentales. Sobre esta cuestión MORAND, C.-A. "Éléments de légistique...", cit., p. 35 (a propósito del contexto suizo); DE MONTALIVET, P. "La «juridicisation» de la légistique. À propos de l'objectif de valeur constitutionnelle d'accessibilité et d'intelligibilité de la loi”, AA.VV. (Drago, R., dir.) La confection de la loi, Paris, 2005, pp. 103 y ss. (sobre el ámbito francés); GARCÍA-ESCUDERO MÁRQUEZ, P. Técnica legislativa y seguridad jurídica: ¿hacia el control constitucional de la calidad de las leyes?, Navarra, 2010, pp. 37, 38 (sobre el caso español).

${ }^{25}$ Tenemos en cuenta en este punto a TAMANAHA, B. Z. “A Concise Guide...," cit., p. 25.
} 
eficiencia, aunque insuficiente para hablar de justicia, tiene un lugar reservado en su discurso.

Además de todo lo anterior, debe tenerse también en cuenta que la justificación de la formación en legística se incrementa a la luz de determinadas situaciones que apreciamos en el panorama jurídico. Podemos referirnos, por ejemplo, a la legislación simbólica, es decir, aquella que carece de los recursos necesarios para que los objetivos que pretende lograr puedan tener lugar realmente ${ }^{26}$. Para evitar resultados de este tipo es necesaria una formación que, además de incidir en la redacción, tenga en cuenta también todo aquello que debe pasar por el tamiz de la racionalidad normativa, tanto en su dimensión formal como material. En este sentido, podemos considerar, por ejemplo, las labores encaminadas a evitar la legislación de mala calidad, causante de más males que de bienes, o aquellas dedicadas a proponer soluciones más económicas en base a la evaluación de costes y beneficios; algo que, de nuevo, en nuestros días es más necesario que nunca, habida cuenta de la contracción de los presupuestos estatales ${ }^{27}$.

\section{III. ¿DÓNDE Y CUÁNDO FORMAR?}

De algún modo, en el desarrollo expuesto hasta el momento ya se ha bosquejado la respuesta a los interrogantes que encabezan este espacio. Conviene, no obstante, abundar en la cuestión mediante el recurso a las experiencias desarrolladas a propósito del tema que nos interesa. Tal ejercicio nos descubrirá numerosos detalles acerca del engranaje de la formación desde el punto de vista institucional, dándonos a conocer también otros posibles cauces para desarrollar aquella.

La puesta en práctica de la formación en redacción de textos legales tiene una importante trayectoria en el ámbito de la Commonwealth. Ya en 1974 encontramos la creación de un Instituto de redacción normativa en Australia. En este centro se llevaron a cabo cursos de formación e instrucción sobre redacción normativa, asistencia a otros países en la formación de personal de redacción de normas, investigaciones sobre la materia, etc. ${ }^{28}$ También desde el mismo año, la Commonwealth Secretariat ha venido organizando cursos de formación para establecer un núcleo de personas bien preparadas, capaces de formar a su vez a otras en sus lugares de procedencia. Los cursos se organizan en colaboración con diferentes Gobiernos ${ }^{29}$. Un ejemplo de ello lo ofrece el Máster en redacción legislativa organizado por la institución citada, en colaboración con la Universidad de Indias Occidentales ${ }^{30}$. En la actualidad, no obstante, uno de los exponentes más relevantes en la cuestión que tratamos es el Institute of Advanced Legal

\footnotetext{
${ }^{26}$ Nos hemos ocupado de esta cuestión en CENTENERA SÁNCHEZ-SECO, F. "La crisis de la ley en Luigi Ferrajoli: algunas consideraciones desde la teoría de la legislación", Cuadernos Electrónicos de Filosofía del Derecho, 26, 2012, pp. 302-306.

${ }^{27}$ MORAND, C.-A. "Éléments de légistique...., cit., p. 35.

${ }^{28}$ KELLY, D. "The Victorian Experience of Plain Drafting", AA.VV. Law Commission. Legislation and its interpretation. Discussion and seminar papers, New Zealand, 1989, p. 66.

${ }^{29}$ CRABBE, V. "The ethics of legislative drafting", Commonwealth Law Bulletin, 36, 1, 2010, p. 12. Sobre un curso breve en la Universidad de Guyana puede verse WEBSTER, R. "Teaching legislative drafting: reflections on the Commonwealth Secretariat Short Course", Commonwealth Law Bulletin, 36, 1,2010 , pp. 43 y ss.

${ }^{30}$ CRABBE, V. "The ethics...”, cit., p. 12; WEBSTER, R. “Teaching legislative drafting...”, cit., p. 41.
} 
Studies de la Universidad de Londres. En este centro se imparte un máster $^{31}$ y se desarrolla una clínica de redacción legislativa ${ }^{32}$. Quienes participan en ella se forman sin la presión de un ambiente profesional y bajo la supervisión de personal experto. Con su labor llevan a cabo al mismo tiempo una actividad pro bono, que beneficia a la clientela real que solicita sus servicios ${ }^{33}$.

Como puede verse, las experiencias desarrolladas parten bien de instituciones, bien de universidades o de centros adscritos a universidades, etc. Conviene señalar además que se trata de propuestas desarrolladas en el marco del postgrado; algo que no debe extrañar si se tienen en cuenta testimonios procedentes de diferentes ámbitos, que confirman la idoneidad de este espacio académico para llevar a cabo los estudios que nos ocupan ${ }^{34}$.

Además de la relación de opciones que se percibe en el desarrollo anterior, también debe hacerse mención de los congresos organizados por instituciones como la Commonwealth Association of legislative Counsel, en colaboración con cada Commonwealth Law Conference ${ }^{35}$, o desde el punto de vista regional el Canadian Institute for Administration of Justice, o el Australasian Parliamentary Counsel's Committee $^{36}$.

Las prácticas desarrolladas en el contexto anterior no son desde luego exclusivas de la Commonwealth, al menos por lo que respecta a las décadas más recientes. El entorno europeo está ofreciendo también numerosas experiencias interesantes. Como en el caso recientemente expuesto, en este también se pueden apreciar iniciativas universitarias, institucionales o incluso mixtas, si bien es cierto que en varias ocasiones la formación ha llegado en primer lugar a través del ámbito público y tiempo después por la vía universitaria ${ }^{37}$. Instituciones dedicadas a la formación que nos interesa pueden encontrarse en Suiza, Países Bajos, Francia, Suecia o Portugal ${ }^{38}$. Lo cierto es que las

\footnotetext{
${ }^{31}$ WEBSTER, R. "Teaching legislative drafting...", cit., pp. 41 y 42.

32 Se trata de un formato plenamente integrado en la educación legal estadounidense. Sobre ello MARCELLO, D. A. "Legislative Learning...", cit., pp. 77, 78.

33 XANTHAKI, H. (2014), Drafting Legislation..., cit., p. 361. Pueden verse contenidos concretos en UNIVERSITY OF LONDON. Sir William Dale Legislative Drafting Clinic. Disponible en: http://ials.sas.ac.uk/postgrad/LDclinic/LDclinic.htm. Fecha de consulta: 24/04/2016.

34 XANTHAKI, H. Drafting Legislation..., cit., p. 359, considera que este es el ámbito por excelencia para desarrollar la tarea que nos interesa, aunque pueden incluirse algunos aspectos en los estudios ordinarios. En la misma línea WRONKOWSKA S. y GWIZDZ, A. "Poland", AA.VV. (Karpen, U., ed.) Legislation in European Countries, Baden-Baden, 1996, p. 344.

${ }^{35}$ MORAN, E. "Foreword", Commonwealth Law Bulletin, 36, 1, 2010, p. 5.

${ }^{36}$ MORAN, E. "Foreword", cit., p. 5.

37 TAVARES DE ALMEIDA, M. "Country presentations: Portugal", AA.VV. (Mader, L. y Moll, C., ed.) The Learning Legislator, Baden-Baden, 2009, p. 178. El interés que se observa en este contexto por la redacción legislativa hacia la década de los setenta, probablemente se deba, al menos en parte, a la apertura de las facultades de derecho alemanas a las ciencias sociales. Sobre ello MORAND, C.-A. "Éléments de légistique...", cit., p. 36. Desde la perspectiva de la metódica de la legislación, Caupers se refiere a las preocupaciones que surgen en el momento indicado por la toma de decisiones públicas. CAUPERS, J. "Relatório sobre o programa...", cit., p. 18.

38 Desde una perspectiva general puede consultarse KARPEN, U. "Improving Democratic Development...", cit., p. 161. Sobre el entorno suizo es interesante el trabajo de MADER, L. "Legistic Training...", cit., pp. 49-53, donde se hace referencia a las actividades desarrolladas por instituciones como la Swiss Association of legislation, o el Seminar of the Swiss Federal Office of Justice. Sobre el ámbito neerlandés es recomendable el estudio de VAN LOCHEM, P. J. P. M. "The Dutch Academy...", cit., pp. 64 y ss., que se refiere a la Academy for Legislation. A propósito del contexto francés puede consultarse DUPRAT, J.-P. "The French »learning« legislator...”, cit., p. 160, que considera los cursos
} 
experiencias que se desarrollan en estos entornos son sumamente interesantes. Como ejemplo, puede citarse la neerlandesa. En este contexto la Academy for Legislation ha implementado un programa de dos años considerado máster post-universitario. Tiene carácter teórico y práctico, y se desarrolla en la institución citada y en un ministerio (o bien en el Council of State). Además, se realizan visitas a numerosas instituciones europeas $^{39}$. Siguiendo en el ámbito del postgrado debemos también referirnos a un buen número de estudios universitarios, como por ejemplo los desarrollados en la Facultad de Ciencias Políticas de la Universidad de Pisa ${ }^{40}$, la Facultad de Derecho de la Universidad Nova de Lisboa ${ }^{41}$, la Facultad de Derecho de la Universidad de Lisboa ${ }^{42}$, la Facultad de de Derecho y Administración de la Universidad de Varsovia ${ }^{43}$, la Universidad Nacional de $\mathrm{Kiev}^{44}$, y las de Berna y Lucerna ${ }^{45}$.

Debe señalarse además que el contexto europeo presenta numerosas experiencias en los estudios ordinarios de derecho ${ }^{46}$. A diferencia de lo que acontece en otros ámbitos, donde la legística no parece muy presente en el marco académico citado, en Europa sí es posible localizar interesantes prácticas en aquel, aun cuando no tienen el desarrollo que fuera deseable en todo caso. A propósito de ello, podemos, por ejemplo, referirnos al contexto húngaro. Su currículum universitario en los estudios ordinarios no ha venido haciendo especial hincapié en la enseñanza de legislación y redacción. Quizá una prueba de ello sea que dichas materias no tienen carácter obligatorio en todo caso ${ }^{47}$. Un grado mayor de compromiso parece apreciarse en el contexto polaco, donde a partir de la constatación de la insuficiencia de los estudios ordinarios en derecho, se complementó en la mayoría de las universidades el currículum con una clase dedicada a los principios de creación del derecho ${ }^{48}$. Podemos detenernos también en Suiza, concretamente en la Universidad de Ginebra, que ha ofrecido cursos optativos sobre legística, y en la de Zúrich, donde encontramos un curso optativo en el ámbito de la

impartidos en la Ecole nacional d'administration, o el Institut d'Etudes Politiques de París. Sobre Suecia SVENSSON, P. A. "The Swedish way to create lawful, consistent and uniform legislation - a summary presentation", AA.VV. (Mader, L. y Moll, C., ed.) The Learning Legislator, Baden-Baden, 2009, p. 139, que se refiere a la Division for Legal and Linguistic Draft Revision. En relación al contexto portugués puede consultarse TAVARES DE ALMEIDA, M. "Country presentations...", cit., pp. 178, 179, donde se considera el Instituto Nacional de Administração. Más concretamente, pueden verse las siguientes referencias: AA.VV. A feitura das leis, tomo I, Oeiras, 1986; AA.VV. A feitura das leis, tomo II, Oeiras, 1986.

${ }^{39}$ VAN LOCHEM, P. J. P. M. “The Dutch Academy...”, cit., pp. 64, 65.

${ }^{40}$ Puede verse un resumen de la estructura del mismo en UNIVERSITÀ DI PISA. Master in Scienza della legislazione. Disponible en: http://www.altalex.com/index.php?idnot=3833. Fecha de consulta: 24/04/2016.

${ }^{41}$ BLANCO DE MORAIS, C. Manual de Legística. Criterios Científicos e Técnicos para Legislar Melhor, [s. 1.], 2007, pp. 635-638; TAVARES DE ALMEIDA, M. "Legista...”, cit., p. 41.

42 TAVARES DE ALMEIDA, M. "Country presentations...”, cit., p. 179; TAVARES DE ALMEIDA, M. “Legista...”, cit., p. 41.

${ }^{43}$ GWIZDZ, A. "Organization of the Post-Graduate Course...", cit., p. 106; WRONKOWSKA S. y GWIZDZ, A. "Poland", cit., p. 344.

44 SEMENOVA, N. "Teaching the Learning Legislator in Ukraine: A country presentation", AA.VV. (Mader, L. y Moll, C., ed.) The Learning Legislator, Baden-Baden, 2009, p. 151.

${ }^{45}$ MADER, L. "Legistic Training...", cit., p. 48.

${ }^{46}$ CAUPERS, J. "Relatório sobre o programa...", cit., p. 62, considera que la metódica de la legislación puede darse en todos los cursos de este ámbito académico.

${ }^{47}$ DRINÓCZI, T. “Quality Drafting...”, cit., p. 168.

${ }^{48}$ GWIZDZ, A. "Organization of the Post-Graduate Course...", cit., p. 108; WRONKOWSKA S. y GWIZDZ, A. "Poland", cit., p. 344. 
educación del derecho público ${ }^{49}$. Interesantes son además los cursos optativos ofertados en la Universidad de Amberes y Bruselas, si bien únicamente tratan algunos aspectos de la legislación ${ }^{50}$; el curso sobre redacción legislativa de la Universidad Nacional de $\mathrm{Kiev}^{51}$; o la asignatura optativa sobre metódica de la legislación de la Universidad Nova de Lisboa ${ }^{52}$. En esta lista podemos incluir también una asignatura sobre redacción de textos legales que impartimos en la Universidad de Alcalá, y que tiene carácter transversal (ello quiere decir que no es obligatoria y que el alumnado de cualquier titulación de la Universidad se puede matricular en ella, en el caso de que tenga interés en la materia $)^{53}$. Al menos por lo que alcanzamos a conocer es un curso innovador en los estudios ordinarios de derecho en el ámbito español.

Siguiendo en la misma parcela académica, quisiéramos llamar también la atención acerca de la siguiente posibilidad. Como es sabido, tras la implementación del Plan Bolonia en el ámbito europeo, el alumnado ha de realizar un trabajo de fin de grado para completar los estudios. Este contexto académico podría suponer también una posible opción para desarrollar la formación que estamos considerando.

Por último, cabe señalar que en el ámbito europeo también se celebran numerosos eventos, que pueden suponer otro cauce más para la formación. La siguiente lista de entidades organizadoras es meramente ejemplificativa: la European Association of Legislation ${ }^{54}$, el Law Moderation Service en el contexto flamenco belga ${ }^{55}$, el Conseil $d^{\prime}$ Etat en Francia ${ }^{56}$, y los Congresos estatales y autonómicos en España ${ }^{57}$.

Es posible que quien nos siga en la lectura tenga en estos momentos en mente la docencia de carácter presencial. Cabría, no obstante, preguntarse si en la cuestión que nos ocupa puede contemplarse la enseñanza a distancia ${ }^{58}$. A nuestro modo de ver su implementación es posible, pero consideramos también que dicha modalidad comprende más complejidad que aquella que pudiera observarse en el entorno presencial. Esta reflexión puede entenderse mejor a la luz de lo que diremos en el apartado dedicado a la metodología.

\footnotetext{
${ }^{49}$ MADER, L. "Legistic Training...", cit., p. 48. En la actualidad, no obstante, al menos algunos de estos cursos son obligatorios. Así, en UNIVERSITÉ DE GENĖVE. Rédaction juridique. Disponible en: http://wadme.unige.ch:3149/pls/opprg/w_det_cours.debut?p_code_cours $=5330 \& p \_p l a n \_i s=0 \& p \_l a n g u e=$ $1 \& p \_$frame $=$N\&p_mode $=$PGC\&p_annee $=2014 \& p \_s u f f i x e=\& p \_g r t r i=12490 . \quad F e c h a ~ d e$ consulta : $24 / 04 / 2016$.

${ }^{50}$ POPELIER P. y VELINDER, V. "Framework for a Country Comparative perspective: Belgium", AA.VV. (Mader, L. y Moll, C., ed.) The Learning Legislator, Baden-Baden, 2009, p. 128.

${ }^{51}$ SEMENOVA, N. "Teaching the Learning Legislator...", cit., p. 151.

${ }^{52}$ CAUPERS, J. "Relatório sobre o programa...", cit., pp. 4-87; BLANCO DE MORAIS, C. Manual de Legística..., cit., p. 638; TAVARES DE ALMEIDA, M. "Country presentations...”, cit., p. 179; TAVARES DE ALMEIDA, M. “Legista...”, cit., p. 41.

53 UNIVERSIDAD DE ALCALÁ. Redacción de Textos Legales. Disponible en: http://www.uah.es/estudios/asignaturas/programas/G61/100076_G61_2015-16.pdf. Fecha de consulta: 30/04/2016.

${ }^{54}$ KARPEN, U. “Improving Democratic Development...", cit., p. 161.

${ }^{55}$ POPELIER P. y VELINDER, V. "Framework...", cit., p. 130.

${ }^{56}$ DUPRAT, J.-P. “The French »learning« legislator...”, cit., p. 160.

${ }^{57}$ AA.VV. III Jornadas de Derecho Parlamentario. La función legislativa de los parlamentos y la técnica de legislar, Madrid, 2000; AA.VV. Legislador i tècnica legislativa. Workshop celebrat al Palau del Parlament el dia 17 de Febrer de 2003, Barcelona, 2003.

${ }^{58}$ Esta opción se considera en CRABBE, V. "The ethics...", cit., p. 12; WEBSTER, R. "Teaching legislative drafting...”, cit., p. 55.
} 


\section{IV. ¿EN QUÉ FORMAR?}

Según algunos estudios, a la hora de plantear un programa de formación dedicado a la creación del derecho es necesario tener en cuenta varias materias jurídicas. Por lo general, desde este punto de vista se hace referencia al derecho constitucional o al derecho administrativo ${ }^{59}$, aunque la lista no es cerrada; en ocasiones también se solicitan, por ejemplo, conocimientos de derecho penal ${ }^{60}$ e incluso podrían considerarse los relativos al derecho procesal. Además, si seguimos, por ejemplo, el modelo del curso de postgrado en legislación que acerca Gwizdz, desarrollado en la Facultad de Derecho de la Universidad de Varsovia, deberá decirse que materias como derecho constitucional o sociología del derecho se encuentran justificadas en el currículum de dicho curso, dado que con ellas se pretende refrescar el conocimiento jurídico del alumnado, tratando así de transmitir los avances en las ciencias y los cambios recientes en el ámbito del derecho constitucional ${ }^{61}$. Debe señalarse, no obstante, que sobre esta cuestión existen diferentes opiniones. Así, por ejemplo, Caupers, profesor que ha impartido la asignatura sobre metódica de la legislación en la Universidad Nova de Lisboa, considera que la compresión de aquella materia no presupone tener conocimientos propios de la ciencia del derecho ${ }^{62}$. A nuestro modo de ver, el discurso en esta cuestión puede venir mediatizado, al menos en parte -de ahí probablemente la diversidad de opiniones-, por aspectos tales como el ámbito académico en el que se pretenda llevar a cabo los estudios, la parcela de análisis o tarea que se quiera abordar, o el nivel de conocimientos que pueda tener el alumnado. Quizá en un primer momento pueda considerarse que quienes cursan legística han de adquirir abundantes conocimientos en materias jurídicas como las que venimos citando. Sin embargo, en base a nuestra experiencia docente podríamos plantear las siguientes reflexiones. Por una parte, puede ser interesante reseñar que, como veremos en breve, la metodología de los estudios en legística no se restringe a la elaboración de una disposición. Puede tratarse simplemente de redactar una oración o un artículo hipotético correctamente, sin que para ello sean necesarios conocimientos jurídicos. Por otra parte, en lo que respecta a la redacción de una norma entendemos que es suficiente con aportar los conocimientos jurídicos que se consideren necesarios en cada momento, para desarrollar la regulación concreta que se pretenda llevar a cabo. Así, por ejemplo, si nuestro alumnado está comenzando los estudios de derecho y pretendemos que redacte la parte de la norma que establece el título competencial habilitante, será necesario abordar los conocimientos que proporciona el ámbito constitucional en la materia en cuestión.

\footnotetext{
${ }^{59}$ Ello es algo característico de diferentes contextos jurídicos. En este sentido puede verse XANTHAKI, H. Drafting Legislation..., cit., p. p. 359; BERGERON, R. C. "The Training of Drafters", AA.VV (Karpen, U. y Delnoy, P., ed.) Contributions to the Methodology of the Creation of Written Law, BadenBaden, 1996, p. 102; MARKMAN, S. C. "Training of Legislative Counsel: learning to draft without Nellie", Commonwealth Law Bulletin, 36, 1, 2010, pp. 27 y 28; PAGANO, R. Introduzione alla legistica. L'arte di preparare le leggi, Milano, 2001, p. 97.

${ }^{60}$ BERGERON, R. C. "The Training of Drafters", cit., p. 102. Por su parte, MORAN, E. "Foreword", cit., p. 4, se refiere a la familiaridad con el proceso penal, en el caso de que tengan que redactarse normas de esta rama del derecho.

${ }^{61}$ GWIZDZ, A. "Organization of the Post-Graduate Course...", cit., pp. 108, 109.

${ }^{62}$ CAUPERS, J. "Relatório sobre o programa...", cit., p. 62.
} 
En cualquier caso, e independientemente de la decisión que se tome en relación a la consideración de materias como las citadas anteriormente, debe decirse que un programa sobre la formación que nos interesa requiere otras propias de la legística. Nos referimos a las que tienen que ver con cuestiones tales como el proceso legal o las convenciones sobre redacción ${ }^{63}$. A continuación presentamos un esquema propuesto por Karpen $^{64}$, que nos puede ayudar a plantear un primer acercamiento a las posibles temáticas a desarrollar:

1. Teoría de la legislación. Hace referencia al concepto de la ley, su evolución y análisis comparado.

2. Analítica de la legislación. Estudia la ley desde la perspectiva de las fuentes del derecho. De este modo, tiene en cuenta las funciones del Estado, la división de poderes y la sistemática de las normas.

3. Táctica de la legislación. Centra la atención en lo que se ha denominado "procedimiento externo de la legislación" y se ocupa de titulares, órganos, organización, procedimientos de legislación, y métodos de influencia en la dirección y producción legislativa.

4. Metódica de la legislación. Se centra en el "procedimiento interno de la legislación" y se ocupa del contenido de las leyes, sus fines, los medios disponibles, y los métodos de evaluación e implementación.

5. Técnica de la legislación. Considera la sistematización y el lenguaje de las leyes, teniendo en cuenta la audiencia de las mismas.

En nuestra opinión, esta lista supera el ámbito que comprende la legística. Al menos así ha de entenderse, si seguimos manteniendo la acepción de aquella que consideramos al comienzo de este estudio, y que nos remite a una dimensión formal, dedicada a la técnica de la legislación con las matizaciones señaladas, y a otra material, que analizada desde la relación expuesta comprendería la metódica de la legislación y una parte de la táctica ${ }^{65}$.

Por lo que hemos tenido oportunidad de ver en nuestro análisis, las experiencias desarrolladas, aun puestas en práctica en diferentes niveles académicos, se conforman

\footnotetext{
${ }^{63}$ MARKMAN, S. C. "Training of Legislative Counsel...", cit., pp. 27 y 28; BERGERON, R. C. "The Training of Drafters", cit., p. 102; XANTHAKI, H. Drafting Legislation..., cit., p. 359; MORAN, E. "Foreword", cit., p. 4; PAGANO, R. Introduzione alla legistica..., cit., p. 97.

${ }^{64}$ Esta propuesta de KARPEN, U. "Zum gegenwärtigen Stand der Gesetzgebungslehre in der Bundesrepublik Deutschland", Zeitschrift für Gesetzgebung, 1, 1986, pp. 5-32 (citado en las referencias siguientes de Atienza y Gomes Canotilho) se recoge en ATIENZA, M. Contribución a una teoría de la legislación, Madrid, 1997, p. 64; GOMES CANOTILHO, J. J. "Relatório sobre programa...", cit., pp. 405-494; CAUPERS, J. "Relatório sobre o programa...", cit., p. 10. En estos casos encontramos desarrollos tanto desde el punto de vista de la investigación (en el primer caso), como desde el docente (en los dos últimos casos). Estos contenidos se abordan además en numerosas obras doctrinales, aunque con diferentes matices y denominaciones. Así, por ejemplo, en ZAPATERO, V. El Arte de Legislar, cit., pp. 76 y ss.; MORAND, C.-A. "Éléments de légistique...", cit., pp. 18 y ss; MARCILLA CÓRDOBA, G. Racionalidad legislativa..., cit., pp. 286 y ss.; MARCILLA CÓRDOBA, G. "Sobre la necesidad de una nueva ciencia de la legislación”, cit., pp. 108 y ss.; MARCILLA CÓRDOBA, G. "Argumentación jurídica y racionalidad legislativa en el Estado Constitucional", Anuario de Filosofía del Derecho, 21, 2004, pp. 345 y ss.; GALIANA SAURA, A. La ley: entre la razón y la experimentación, Valencia, 2008, pp. 39 y ss.; SALVADOR CODERCH, P. "Técnica legislativa y teorías de la regulación", InDret, 2, 2004, pp. 10 y ss.; MADER, L. "Legislative Procedure and the Quality of Legislation", AA.VV. (Karpen, U. y Delnoy, P., ed.) Contributions to the Methodology of the Creation of Written Law, Baden-Baden, 1996, pp. 62 y SS.

${ }^{65}$ Seguimos en este punto a CAUPERS, J. “Relatório sobre o programa...”, cit., p. 11.
} 
en base a los parámetros descritos (las denominaciones, no obstante, varían en los programas), si bien es cierto que no se consideran todos los puntos. Los modelos suelen centrarse -aunque no exclusivamente en todo caso- en las temáticas relativas a la legística, entendida con las precisiones recientemente expuestas, pero a menudo poniendo el énfasis en determinados ámbitos de aquella. A propósito de ello, puede ser interesante reseñar que la titulación de las asignaturas suele ser en numerosas ocasiones indicativa de las parcelas que desarrollan, o bien de su nivel de preponderancia. Podemos, por ejemplo, fijarnos en la experiencia desarrollada en la Facultad de Derecho de la Universidad Nova de Lisboa, donde se ha venido ofertando una asignatura en la que se presentan contenidos enmarcables en buena medida dentro de la metódica de la legislación. En el programa se tratan puntos como la iniciativa e impulso legislativo (que, no obstante, entendemos que formaría parte de la táctica de la legislación), la respuesta a las cuestiones de para qué la ley, si la ley en cuestión puede alcanzar los objetivos que se pretenden, los posibles mecanismos de regulación, la conveniencia del tiempo para legislar, quién debe legislar, qué ley hacer, o la claridad y la precisión en la forma (estos dos últimos aspectos entendemos que quedarían enmarcados en el ámbito de la técnica de la legislación). Además, en este modelo se desarrollan dos tipos de evaluación interesantes: la prospectiva (que tiene en cuenta la determinación de las condiciones adecuadas para producir los efectos deseados, las reacciones de las personas a las que se destina la ley y el análisis coste-beneficio), y la retrospectiva (que analiza los efectos de la ley en la sociedad, tratando de mejorar la acción estatal con más eficacia, eficiencia y/o atenuación de los efectos negativos) ${ }^{66}$.

Podemos considerar además la propuesta de Karpen, que aun cuando dedica una parte a la metódica, considera de manera más pormenorizada aspectos de otros puntos del esquema, de entre los que quisiéramos destacar la técnica de la legislación. A nuestro modo de ver, con ello el modelo que nos ocupa muestra un acercamiento a las tradiciones de Inglaterra y Estados Unidos, caracterizadas por su especial preocupación por la redacción de normas ${ }^{67}$. En esta línea, el proyecto en cuestión incluye en su programa aspectos tales como la estructura de la oración, el tema de la misma, el tiempo, modo, número y voz verbales, la consistencia, la brevedad, la puntuación, las mayúsculas, las expresiones a evitar, las enumeraciones, las tabulaciones, el lenguaje de género, etc. ${ }^{68}$ Con una perspectiva parecida se desarrolla el programa del curso que impartimos en la Universidad de Alcalá, donde también tienen un papel importante los

\footnotetext{
${ }^{66}$ CAUPERS, J. "Relatório sobre o programa...", cit., pp. 13, 15, 30-61; BLANCO DE MORAIS, C. Manual de Legística..., cit., p. 638.

${ }^{67}$ La doctrina que consideramos en este estudio, procedente de los contextos en cuestión, ofrece prueba de ello. No obstante, en este punto no quisiéramos renunciar a la cita de Bentham, que da muestra de una tradición dilatada (probablemente una de las obras más paradigmáticas sea BENTHAM, J. Nomografía o el arte de redactar leyes, Madrid, 2004), o a la de un clásico reeditado en los años recientes: THORNTON, G. C. Thornton's Legislative Drafting, [London], 2013. Por otra parte, ofrecen también muestra de lo que decimos un buen número de manuales que contemplan numerosos aspectos relativos a la redacción legislativa. Como muestra, la siguiente referencia: THE OFFICE OF LEGISLATIVE LEGAL SERVICES. Colorado Legislative Drafting Manual. Disponible en: http://tornado.state.co.us/gov_dir/leg_dir/olls/LDM/OLLS_Drafting_Manual.pdf. Fecha de consulta: 10/05/2016. Conviene señalar, no obstante, que el interés por la redacción normativa también aparece de modo muy relevante en manuales desarrollados en contextos diferentes. Podemos citar, por ejemplo, la siguiente referencia, que reserva una extensión muy considerable al tema en cuestión: MARTINO, A. A. Manual de Técnica Legislativa. Disponible en: http://www.antonioanselmomartino.it/Digesto/manual.html. Fecha de consulta: 10/05/2016.

${ }^{68}$ KARPEN, U. “Law Drafting...”, cit., pp. 16, 17.
} 
aspectos lingüísticos, tales como la construcción de oraciones y párrafos, la ambigüedad, la vaguedad, la claridad, la brevedad, etc. ${ }^{69}$

Conviene, no obstante, apuntar que algunos modelos presentan una especial fijación en determinados temas, que al menos en parte puede obedecer a características particulares del contexto jurídico en el que se desarrolla la formación, o bien a circunstancias que han afectado de una manera importante a aquel. Un ejemplo en el que pueden advertirse aspectos que traen causa de las particularidades del ámbito en el que se desarrolla la formación, lo encontramos en una experiencia desarrollada hace ya tiempo en la Universidad de Ottawa. Se trata de un programa compuesto por seminarios donde se abordan materias que ya conocemos (la redacción legislativa, las convenciones sobre dicha cuestión, el proceso legislativo, o la aplicación del derecho constitucional al proceso de elaboración de leyes), pero también otra que podría resultar sugerente: nos referimos al estudio de la legislación delegada ${ }^{70}$. Por otra parte, nos interesa considerar el programa desarrollado por la Academy for Legislation neerlandesa, donde podemos localizar los siguientes puntos $^{71}$ :

1. Teoría de la legislación, procedimiento legislativo y técnica legislativa.

2. Derecho administrativo, derecho constitucional $y$, de una forma especial, derecho europeo.

3. Temas de carácter político y social.

Como puede verse, algunas de las materias que encontramos en este modelo forman parte de los estudios ordinarios de derecho. Sin embargo, su inclusión en esta ocasión tiene su razón de ser en el hecho de profundizar en dichas disciplinas, relacionándolas directamente con el tema de la legislación ${ }^{72}$. Por otra parte, queremos referirnos a la especial fijación de este programa en el derecho europeo, algo que, no obstante, no es exclusivo del mismo ${ }^{73}$. Ello obedece a una sentencia del Tribunal de Justicia de la Unión Europea, que dejó sin efecto legislación belga por pasar por alto las reglas de procedimiento europeas recogidas en la directiva 83/189, y que tuvo impacto en el ámbito neerlandés, dado que al parecer, se diagnosticaron casos parecidos alli ${ }^{74}$.

Por lo que respecta al entorno europeo, la inclusión en el programa de materias como la recientemente expuesta parece sugerente. Desde una perspectiva más general, incluso podría considerarse el tratamiento de didácticas no eminentemente jurídicas, sino más bien de carácter transversal (sociología o economía, por ejemplo). No obstante, a nuestro modo de ver $-\mathrm{y}$, de nuevo, tomando como base nuestra experiencia docente- sería suficiente con transmitir los conocimientos de las materias en cuestión que fueran necesarios, con vistas desarrollar la labor que se pretenda llevar a cabo en el aula.

\footnotetext{
${ }^{69}$ UNIVERSIDAD DE ALCALÁ. Redacción de Textos Legales, cit., pp. 4, 5.

${ }^{70}$ BERGERON, R. C. "The Training of Drafters", cit., pp. 104 y 105.

${ }^{71}$ VAN LOCHEM, P. J. P. M. "The Dutch Academy...”, cit., p. 65.

72 VAN LOCHEM, P. J. P. M. “The Dutch Academy...”, cit., p. 65.

${ }^{73}$ Puede, por ejemplo, apreciarse también en el en el programa desarrollado por el Instituto Nacional Portugués de Administración Pública. Sobre ello TAVARES DE ALMEIDA, M. "Country presentations...”, cit., p. 179.

${ }^{74}$ VAN LOCHEM, P. J. P. M. “The Dutch Academy...”, cit., p. 63.
} 


\section{V. ¿CÓMO FORMAR?}

\section{Conocimientos y habilidades}

La puesta en práctica de programas como los expuestos conlleva la transmisión de conocimientos, pero nos interesa mucho hacer notar que no exclusivamente. Ciertamente, con la labor citada pueden aprenderse aspectos jurídicos de un determinado contexto, modelos, técnicas de redacción, reglas de sintaxis, etc. ${ }^{75}$ Sin embargo, es necesario algo más. Cuando alguien quiere aprender a componer música, antes debe haber cursado solfeo y armonía, entre otros estudios, pero el conocimiento del lugar que ocupan las notas en el pentagrama y de las diferentes escalas no garantiza que se pueda componer música. Para ello es preciso entrenar las habilidades correspondientes para hacer posible esta última labor. Algo parecido pensamos que sucede con el desarrollo de las tareas propias de la formación que nos ocupa, una parcela en la que además de transmitir conocimientos es necesario entrenar en habilidades en diferentes ámbitos. A propósito de lo que venimos diciendo, tiene mucho sentido que consideremos en este momento la concepción de la labor legislativa como arte. Ciertamente, la actividad que centra nuestra atención tiene una parte técnica o científica si se quiere, que remite a la existencia de determinadas reglas ${ }^{76}$, pero no se agota en ello. La tarea en cuestión supone algo más, pues como vamos a ver quien legisla no actúa según patrones fijos ${ }^{77}$. En su trabajo debe verse también una parte importante de $\operatorname{arte}^{78}$, que conlleva solucionar cuestiones partiendo de guías, pero no de guiones $^{79}$, y que como todo arte tiene su práctica y especulación ${ }^{80}$. Tal planteamiento subraya la relevancia que tiene el entrenamiento en habilidades a propósito del contexto que nos interesa.

Antes de continuar, quisiéramos apuntar que el proceso de creación del derecho tiene unas características que marcan diferencias con respecto al ámbito judicial, donde se desarrollan la aplicación e interpretación normativa. Aquellos aspectos han de tenerse en cuenta a la hora de planificar el entrenamiento en habilidades y las prácticas a realizar, si se pretende ofrecer una formación adecuada en el contexto que nos interesa (ello, no obstante, no impide reconocer recomendaciones generales aplicables a ambas parcelas $^{81}$, o las aportaciones que puede reportar la argumentación judicial en el contexto que nos interesa ${ }^{82}$ ). Las diferencias entre el ámbito de la aplicación e

\footnotetext{
${ }^{75}$ BERGERON, R. C. "The Training of Drafters", cit., p. 104.

${ }^{76}$ XANTHAKI, H. Drafting Legislation..., cit., pp. 354, 355.

${ }^{77}$ FULLER, L. L. "What the Law Schools Can Contribute...", cit., p. 195.

${ }^{78}$ XANTHAKI, H. Drafting Legislation..., cit., pp. 355-359; KARPEN, U. "Resumé”, AA.VV. (Karpen, U., ed.) Legislation in European Countries, Baden-Baden, 1996, p. 487; BERGEL, J.-L. "The Drafting of the Norm", AA.VV. (Karpen, U. y Delnoy, P., ed.) Contributions to the Methodology of the Creation of Written Law, Baden-Baden, 1996, pp. 49, 50; NAMPOOTHIRY, N. K. "The role of Parliamentary Counsel in legislative drafting", Commonwealth Law Bulletin, 36, 1, 2010, p. 60.

${ }^{79}$ XANTHAKI, H. Drafting Legislation..., cit., p. 360.

${ }^{80}$ ZAPATERO. V. "El arte ilustrado de legislar", Bentham, J. Nomografía o el arte de redactar leyes, Madrid, 2004, pp. XVII, XVIII.

${ }^{81}$ Así, por ejemplo, en lo que respecta a la claridad del lenguaje. ATIENZA, M. Contribución..., cit. p. 96.

${ }^{82}$ Sobre ello puede consultarse ATIENZA, M. Contribución..., cit. p. 96; MARCILLA CÓRDOBA, G. “Argumentación jurídica y racionalidad legislativa...", cit., p. 342; MARCILLA CÓRDOBA, G.
} 
interpretación, y aquel dedicado a la creación del derecho pueden observarse, por ejemplo, en los siguientes aspectos ${ }^{83}$ : el margen de decisión sobre los problemas que se tratan (referenciado por el marco normativo en el primer ámbito y con opciones de decisión más amplias y creativas en el segundo, contextualizado además en la parcela de las políticas públicas); el planteamiento del razonamiento básico (subsunción en el primer escenario y balance de razonamientos en el segundo) el objeto de análisis (la sentencia en el primer contexto, y el proceso legislativo y la redacción de la norma en el segundo); o el carácter de los casos que se consideran (concreto en el primer escenario y general en el segundo; en terminología de Bobbio, de un lado normas particulares y concretas, y de otro normas generales y abstractas ${ }^{84}$ ). Cabría añadir además que de estas particularidades podrían derivarse otras, como por ejemplo las peculiaridades en la estructura, a propósito de la tipología de textos citada.

Si nos detenemos en el ámbito de la redacción propiamente dicha, habremos de reconocer que en él la transmisión y adquisición de conocimientos acerca de cómo escribir correctamente no garantiza un buen resultado. Es más, en la práctica es posible que aunque se tenga una idea clara en mente, su reflejo en el papel de forma clara y precisa pueda no ser fácil ${ }^{85}$. Desde este punto de vista son numerosas las tareas que deben abordarse. La siguiente lista ofrece muestra de ello: capacidad de enfocar, con la que se identifica el núcleo de la cuestión a desarrollar y las formas en las que los detalles se relacionan con dicho núcleo ${ }^{86}$; fluidez en la escritura, que facilita un trabajo rápido, elegante y preciso $^{87}$; capacidad para desarrollar ideas ${ }^{88}$; capacidad para escribir de forma coherente, conectando de forma lógica las partes del trabajo, tanto formal como materialmente ${ }^{89}$; y capacidad para tener una mente ordenada, algo que redundará

\footnotetext{
“Argumentación legislativa y teoría estándar...", cit., pp. 511 y ss. En nuestra modesta opinión, no obstante, podrían plantearse varios comentarios. De un lado, queda pendiente la tarea de concretar de forma pormenorizada las aportaciones a las que nos referimos. De otro lado, aun reconociendo lo anterior pensamos que existen diferencias que conviene considerar. Puede pensarse, por ejemplo, que en la redacción de una norma se tienen en cuenta los aspectos lingüísticos, y que estos también son objeto de atención en algunas sentencias del Tribunal Constitucional. Cabría considerar, no obstante, que nos estamos refiriendo a un tribunal concreto, que no en todas sus sentencias se contempla la cuestión citada y que cuando es así, se entiende que el principio de seguridad jurídica se infringe únicamente en casos que den lugar a una incertidumbre insuperable. En el proceso legislativo, sin embargo, la atención en las cuestiones lingüísticas es ineludible en todo caso durante la redacción, y la preocupación en esta parcela alcanza al nivel recientemente señalado, pero va más allá, pues lo que se pretende en este ámbito es obtener las cotas más altas posibles de calidad legislativa. Hemos tratado puntualmente la cuestión en CENTENERA SÁNCHEZ-SECO, F. "La claridad legislativa en el pensamiento de Lon L. Fuller: un análisis desde la teoría de la legislación”, Anales de Derecho, 1, 2015, pp. 19 y ss.

${ }^{83} \mathrm{La}$ siguiente relación y otros aspectos más pueden localizarse en MARCILLA CÓRDOBA, G. “Argumentación legislativa y teoría estándar...", cit., pp. 507, 508. Esta lista se plantea con carácter general. En función de determinados casos deberían reconsiderarse los contenidos expuestos (pensemos, por ejemplo, en los contratos).

${ }^{84}$ BOBBIO, Teoría General del Derecho, Bogotá, 2005, p. 132.

${ }^{85}$ CRABBE, V. "The ethics...", cit., p. 13. Sobre ello también GWIZDZ, A. "Organization of the PostGraduate Course...", cit., p. 107.

${ }^{86}$ STARK, J. The Art of the Statute, Littleton, 1996, p. 103.

${ }^{87}$ STARK, J. The Art of the Statute, cit., pp. 103.

${ }^{88}$ STARK, J. The Art of the Statute, cit., pp. 103 y 104; CRABBE, V. “The ethics...”, cit., p. 13.

${ }^{89}$ STARK, J. The Art of the Statute, cit., p. 104. En este ámbito entendemos que pueden entrar en juego cuestiones estilísticas, que determinan, por ejemplo, el orden concreto de la estructura gramatical de la frase. Sobre esta cuestión BERGEL, J.-L. "The Drafting of the Norm”, cit., p. 50.
} 
en un trabajo más eficiente ${ }^{90} \mathrm{y}$ ajustado a las condiciones temporales que acompañan a la actividad legislativa ${ }^{91}$.

Como podrá imaginarse, la adquisición de habilidades como las señaladas pasa obligatoriamente por la práctica. Sin embargo, esta circunstancia no se aprecia únicamente en las parcelas recientemente tratadas. Aun teniendo los conocimientos lingüísticos necesarios, e incluso habiendo cultivado las habilidades pertinentes en este ámbito, es necesario tener también en cuenta que las directrices a seguir en la tarea que nos está ocupando no se aplican conforme al criterio de todo o nada. A propósito de ello, quizá resulte ilustrativo volver la mirada al pensamiento de Fuller, que se refería a la claridad como un desiderátum ${ }^{92}$. Con ello quería decir que, por ejemplo, debe tenderse a la claridad normativa, aun sabiendo que en numerosas ocasiones los niveles de aquella deberán ceder a favor de otros aspectos, que también han de tenerse en cuenta por parte de quien redacta normas. Son varios los ámbitos que podrían considerarse a propósito de lo que decimos, por ejemplo, el político. Es cierto que en la redacción normativa debe aspirarse a la mayor claridad y precisión posibles, pero también lo es que en ocasiones la vaguedad puede constituir un recurso interesante para satisfacer determinadas necesidades políticas ${ }^{93}$. Es precisamente el personal de redacción el que ha de buscar un comedido equilibro en tareas como esta, capaz de satisfacer en lo posible -o de regular el nivel de déficit de- las directrices de carácter lingüístico, pero combinándolas también con lo que se solicita desde otros niveles de racionalidad; porque no debe olvidarse que quien redacta trabaja con el personal del ámbito en el que se gesta la iniciativa legislativa.

La circunstancia recientemente expuesta nos introduce además en otro contexto para el que también es necesario tener determinadas habilidades, que conviene adquirir con la formación. En este sentido, algunos estudios han constatado que quien redacta ha de tener capacidad para analizar las directrices políticas y entender lo que se está pidiendo con ellas, preguntar en el caso de que se considere oportuno con el fin de clarificar los objetivos, investigar las posibles opciones disponibles, administrar bien el tiempo, trabajar en equipo, usar tecnología, saber gestionar los conflictos, etc. ${ }^{94}$ Además de lo anterior, si ponemos el foco de atención en los sistemas de redacción descentralizada, sería necesario formar principalmente en habilidades relacionadas con

\footnotetext{
${ }^{90}$ STARK, J. The Art of the Statute, cit., p. 104; CRABBE, V. "The ethics...", cit., p. 13; MORAN, E. "Foreword", cit., p. 4 (se refiere a la claridad de pensamiento).

${ }^{91}$ En numerosas ocasiones no se tiene tiempo para deliberar ni trabajar sobre los detalles de la regulación, los términos legales o el lenguaje de la norma. Sobre ello KRONGOLD, S. "Writing Laws: making them easier to understand", Ottawa Law Review, 24, 2, 1992, p. 501; ILES, W. "Coment", AA.VV. Law Commission. Legislation and its interpretation. Discussion and seminar papers, New Zealand, 1989, p. 79; KARPEN, U. "Improving Democratic Development...”, cit., p. 157. Sobre lo que puede aportar la habilidad en cuestión a propósito de circunstancias en las que existe presión STARK, J. The Art of the Statute, cit., p. 104.

${ }^{92}$ FULLER, L. L. La moral del derecho, México, 1967, pp. 52, 55. Sobre la cuestión de la ponderación en este ámbito puede verse además MARCILLA CÓRDOBA, G. "Argumentación jurídica y racionalidad legislativa...", cit., pp. 345 y ss.; MARCILLA CÓRDOBA, G. "Argumentación legislativa y teoría estándar...", cit., pp. 511 y ss.; CENTENERA SÁNCHEZ-SECO, F. "Los criterios lingüísticos de las directrices de técnica normativa: una propuesta para la solución de enfrentamientos entre principios", Revista de Llengua i Dret, 58, 2012, pp. 15-34.

${ }^{93}$ ZAPATERO, V. El Arte de Legislar, cit., p. 263; CENTENERA SÁNCHEZ-SECO, F. "Los criterios lingüísticos...”, cit., pp. 26, 27.

${ }^{94}$ MARKMAN, S. C. "Training of Legislative Counsel...", cit., pp. 29, 30, 33, 34, 35; NAMPOOTHIRY, N. K. "The role of Parliamentary Counsel...", cit., p. 59.
} 
la determinación del contenido de la redacción ${ }^{95}$. En este sentido, deberían abordarse cuestiones tales como la definición del problema a resolver, el ajuste de los objetivos que se proponen con respecto a aquel, etc. Desde este punto de vista también sería preciso el entrenamiento en habilidades, pues en la gestión de aspectos tales como los objetivos o los medios a tener en cuenta para resolver un problema dado, conviene realizar un análisis bajo el prisma del "balance de razones" "96.

Si se acepta el planteamiento expuesto, con él debe reconocerse el papel relevante que tiene en este punto la cuestión de la prudencia, y con ello, por lo que a nuestro objeto de estudio respecta, la necesidad de formar también en este sentido en diferentes ámbitos desde una perspectiva práctica.

\section{Posibles metodologías}

Como hemos visto, en la formación que venimos considerando deben transmitirse conocimientos, pero también $-\mathrm{y}$ a nuestro juicio con un protagonismo indudable- habilidades. Desde el punto de vista de la metodología podrían hacerse varias propuestas a propósito de estos dos ámbitos. En primer lugar, en lo que respecta a la transmisión de contenidos, probablemente la primera opción que surja sea la de la clase magistral. A nuestro modo de ver no debe desecharse, pero con ello, y reconociendo al tiempo la utilidad de los manuales y guías que puedan recomendarse ${ }^{97}$, pensamos también que es interesante recurrir a otros recursos que propician la participación del alumnado. Así, por ejemplo, se pueden impartir las clases teóricas de forma dialogada ${ }^{98}$, o bien solicitar al alumnado que prepare un tema, con el fin de que se analice en grupo $\mathrm{y}$ de este modo se puedan aclarar conceptos, jerarquizar conocimientos, etc ${ }^{99}$. Una versión más extendida y detallada de esta propuesta ${ }^{100}$ se puede llevar a cabo del siguiente modo (en esta ocasión, no obstante, lo que se pretende es revisar conocimientos adquiridos durante el curso). También en ella se solicita al alumnado que elabore trabajos, cuya temática se elige por el profesorado encargado del curso, teniendo en cuenta para ello aspectos tales como los conocimientos o intereses de quienes asisten a clase. El alumnado dispone de tiempo suficiente para investigar con los documentos que se crean pertinentes y para, en base a ello, elaborar los trabajos. Una vez realizados se exponen en clase mediante una síntesis oral -no leída-, pero antes deben entregarse al profesorado y a quienes van a asistir a la clase, aproximadamente con ocho días de antelación.

\footnotetext{
95 En el sistema señalado el departamento en el que se gesta la iniciativa legislativa se encarga de la redacción, pero también de determinar el contenido de la norma. Sobre ello TAVARES DE ALMEIDA, M. "Legista...", cit., p. 38. Por ello decimos que principalmente son necesarias habilidades en cuanto a la gestión de este último aspecto. Sobre los sistemas de redacción también ZAPATERO, V. El Arte de Legislar, cit., pp. 215 y ss.

${ }^{96}$ MARCILLA CÓRDOBA, G. “Argumentación legislativa y teoría estándar...”, cit., p. 507.

${ }^{97}$ WEBSTER, R. “Teaching legislative drafting...”, cit., pp. 45, 46; WRONKOWSKA S. y GWIZDZ, A. "Poland", cit., p. 344.

${ }^{98}$ CAUPERS, J. "Relatório sobre o programa...", cit., p. 63

${ }^{99}$ MURO RUIZ, E. "Enseñanza de la técnica legislativa", cit., p. 81, se refiere a ello bajo el rótulo de "razonamiento".

${ }^{100}$ Lo desarrolla GOMES CANOTILHO, J. J. "Relatório sobre programa...”, cit., p. 416, que enmarca la propuesta dentro del formato de seminarios.
} 
Por otra parte, la formación en habilidades también puede llevarse a cabo de acuerdo a metodologías sugerentes. Así, por ejemplo, es interesante la propuesta de redactar una acción o prohibición para mejorar el barrio al que se pertenece. También puede solicitarse la redacción de algunas disposiciones simples, para que sea posible el cambio en un contexto como el recientemente señalado ${ }^{101}$. Los borradores de estos ejercicios, llevados a cabo individualmente, se discuten en la clase con el fin de conectar derecho y política, explorar opciones alternativas para conseguir la misma política, o examinar las implicaciones que conlleva cada opción ${ }^{102}$. Ejercicios de este tipo se pueden realizar a lo largo de todo el curso ${ }^{103}$. Además, en función de las circunstancias puede ser interesante alterar el orden de los objetivos propuestos en experiencias como la recientemente expuesta. Así, en vez de discutir las posibles opciones políticas en la puesta en común final, puede resultar sugerente llevar a cabo este ejercicio en primer lugar ${ }^{104}$ para, a partir de este punto, desarrollar la tarea de redacción.

Otra posible metodología es aquella en la que, además del ejercicio de redactar prohibiciones o disposiciones simples, se solicitan otros consistentes en reescribir normativa antigua, teniendo en cuenta las actuales prácticas de redacción. En esta ocasión se sugiere también el intercambio de trabajos (en los que no se pone el nombre) entre el alumnado, con el fin de determinar las fortalezas y debilidades ${ }^{105}$. Bergeron, que es quien presenta esta experiencia, se refiere también a lo que denomina "instant drafting exercises", que consisten en pequeñas tareas de redacción que el alumnado lleva a cabo en clase en una o dos horas ${ }^{106}$. En la misma línea, aunque con algún detalle interesante, encontramos otra experiencia que incluye también ejercicios de redacción de disposiciones antiguas o mejorables, ejercicios sobre gramática y sintaxis, de redacción de instrucciones, de planificación de una pieza de legislación, etc. Después de llevarlos a cabo, el alumnado se enfrenta a la redacción de una nueva disposición legislativa ${ }^{107}$. En nuestra experiencia docente realizamos varios ejercicios como los recientemente considerados, que van desde la corrección de una oración construida de forma defectuosa, hasta la redacción de una norma ${ }^{108}$.

Prácticas como las propuestas pueden tener lugar a lo largo del curso, y en su desarrollo es posible recurrir a diferentes soportes ${ }^{109}$. En este planteamiento

\footnotetext{
${ }^{101}$ KARPEN, U. "Law Drafting...”, cit., p. 12.

${ }^{102}$ KARPEN, U. "Law Drafting...", cit., p. 12.

${ }^{103}$ KARPEN, U. "Law Drafting...", cit., p. 12.

${ }^{104}$ Mediante lo que Muro Ruiz denomina "mesa redonda" o "promoción de ideas". MURO RUIZ, E. "Enseñanza de la técnica legislativa", cit., pp. 82, 83.

${ }^{105}$ En este ejercicio de intercambio, de alguna forma queda reflejada la recomendación de llevar a cabo revisiones críticas en el entorno de trabajo. Aquellas se realizan por parte de profesionales que no han redactado el texto. Sobre esta cuestión ZAPATERO, V. El Arte de Legislar, cit., pp. 278, 279.

${ }^{106}$ BERGERON, R. C. "The Training of Drafters", cit., p. 105.

${ }^{107}$ WEBSTER, R. "Teaching legislative drafting...”, cit., p. 49.

108 Desarrollamos de forma más detenida algunas de las prácticas que planteamos en clase en CENTENERA SÁNCHEZ-SECO, F. "Recursos para formar...", cit., pp. 136-148.

${ }^{109}$ Buena parte de las experiencias anteriores entendemos que están haciendo referencia al trabajo con ejercicios escritos en papel. En algún caso se recomienda el uso de diapositivas o transparencias. Con estos recursos se puede, por ejemplo, poner en primer lugar una diapositiva con una redacción defectuosa, para después proyectar otra con la versión correcta. Por otra parte, las transparencias ofrecen la opción de que alumnado y profesorado puedan participar en el desarrollo y mejora de la redacción. Sobre ello DICK, R. C. Legal Drafting..., cit., pp. 259, 260. En nuestros días, pensamos que las opciones que ofrecen los procesadores de textos (que se pueden proyectar en el aula) cumplen las mismas funciones que los recursos anteriores. WEBSTER, R. "Teaching legislative drafting...", cit., pp. 45, 50, se refiere al PowerPoint a propósito de la proyección de instrucciones o de modelos de solución.
} 
probablemente sea conveniente implementar un nivel de dificultad ascendente, a medida que van avanzando las clases. Así, por ejemplo, el primer ejercicio puede consistir en plantear la redacción de una regulación para solucionar un problema del barrio al que se pertenece, a continuación se pueden solicitar ejercicios más complicados donde se tengan en cuenta las directrices de redacción, para después desarrollar una práctica más compleja, tomando en consideración la normativa vigente y la jurisprudencia ${ }^{110}$. En todo caso, entendemos que a la hora de evaluar el nivel de dificultad resulta ineludible tener en cuenta el ámbito académico en el que se desarrolla el programa. En base a ello, incluso podría ser conveniente recurrir a grados más propedéuticos que los considerados hasta este momento. Así, por ejemplo, si se trata de una asignatura que se cursa en los primeros años de los estudios ordinarios, probablemente lo más apropiado sea comenzar con cuestiones lingüísticas de tipo general para, a partir de aquí, abordar aquellas y otras nuevas a propósito del ámbito legislativo ${ }^{11}$. Por otra parte, además de tener en cuenta la gradación en lo que respecta a la dificultad, conviene destacar también el papel que ha de desempeñar el profesorado en esta metodología. Su supervisión, tutoría y retroalimentación es a nuestro juicio esencial para que puedan obtenerse buenos resultados.

Como venimos diciendo en este estudio, la práctica de la legística no abarca únicamente la labor de redactar, sino que también tiene en cuenta la que ha de llevarse a cabo en conjunción con el ámbito político. Decíamos anteriormente, basándonos en estudios desarrollados en el marco de la Commonwealth, que quienes redactan han de entender bien las directrices que les llegan, tener habilidades para preguntar, etc. En esta línea se ha propuesto la metodología del role playing, en la que una persona puede adoptar el papel de quien establece las directrices y otra el de quien redacta la norma ${ }^{112}$. No obstante, si centramos la atención en el sistema de redacción descentralizado, puede resultar muy sugerente la siguiente dinámica desarrollada en el ámbito portugués por Caupers. Con ella, de algún modo se ofrece una percepción más pormenorizada de las prácticas que consideramos anteriormente, relativas a las labores a realizar desde el ámbito político. En la experiencia en cuestión se enfrentan posturas a propósito del planteamiento de una norma. Concretamente, se trata de preparar una ley imaginaria sobre una materia determinada. A partir de aquí, el alumnado se divide en dos grupos, uno representa el papel del legislativo, que prepara la ley y debate todas las cuestiones a tener en cuenta, y otro desempeña una función concreta en cada una de las fases del proceso legislativo, dedicándose por ejemplo a defender la inutilidad de la ley, poner en duda los objetivos pretendidos, abogar por los intereses supuestamente lesionados con la nueva ley, etc. En la última clase el primer grupo presenta su propuesta justificada, y el otro considera alternativas o justificaciones para rechazar la ley ${ }^{113}$. Nos encontramos, en definitiva, ante una metodología sugerente para formar en metódica de la legislación.

En este espacio pensamos que también conviene dedicar unas líneas a las propuestas de ejercicios para fin de curso. Una posible opción puede ser solicitar al alumnado que reescriba algunas de las disposiciones que ya se redactaron como

\footnotetext{
${ }^{110}$ MURO RUIZ, E. "Enseñanza de la técnica legislativa", cit., p. 82, se refiere a ello con la expresión case meted.

${ }^{111}$ Esta es la rutina que seguimos en UNIVERSIDAD DE ALCALÁ. Redacción de Textos Legales, cit., pp. 4,5 .

112 MARKMAN, S. C. "Training of Legislative Counsel...", cit., p. 36. Sobre este recurso también WEBSTER, R. "Teaching legislative drafting...", cit., p. 52.

${ }^{113}$ CAUPERS, J. "Relatório sobre o programa...", cit., pp. 65, 66.
} 
ejercicios, y que lleve a cabo uno nuevo escogido por el profesorado encargado del curso $^{114}$. Una práctica más compleja es aquella que presenta un examen compuesto de dos partes. En la primera de ellas ha de redactarse un proyecto de ley corto y en la segunda deben responderse cuestiones de carácter teórico, tanto directas como de discusión $^{115}$. Otra alternativa puede ser que el alumnado presente al final del curso un trabajo de unas 25-30 páginas, sobre cuestiones relativas a la formación que nos ocupa. Para realizar este ejercicio se puede solicitar también la elaboración de una norma sobre un tema determinado ${ }^{116}$. Sería además interesante combinar esta última opción con otra tarea desarrollada con carácter previo, en la que se discuta el contenido de la norma. Según este modelo, que es el que venimos desarrollando en nuestra experiencia docente, a lo largo del curso se analizan aspectos tales como el problema en cuestión, sus posibles causas y cauces de resolución, y posteriormente se va redactando una norma capaz de vertebrar desde el punto de vista jurídico todo lo anterior ${ }^{117}$. De nuevo en estas prácticas, a la hora de establecer el nivel de complejidad convendrá tener en cuenta el contexto académico en el que se desarrolla el curso.

Quisiéramos dejar también algunas reflexiones sobre la posibilidad de implementar la formación que nos ocupa en los trabajos de fin de grado. Al menos por lo que llegamos a conocer, no tenemos noticia de prácticas en este ámbito. Por ello, desarrollamos las siguientes líneas en base a nuestra experiencia docente. En los trabajos de esta clase que venimos tutorizado la rutina que seguimos es la expuesta en la parte final del párrafo anterior, si bien en esta ocasión recurrimos a numerosas tutorías para considerar las materias y tareas del esquema planteado. En base a la experiencia adquirida podemos decir que la práctica en cuestión suele resultar sugerente para el alumnado, pero al mismo tiempo quizá conlleve cierta complejidad para aquel. La razón de ello pensamos que, al menos en parte, debe buscarse en las circunstancias que consideramos al comienzo de este trabajo: el sistema de formación jurídica actual se centra principalmente en la interpretación y aplicación del derecho. Por tanto, quien elabore un trabajo de fin de grado en la línea expuesta tendrá que adquirir conocimientos y habilidades apenas trabajadas en su trayectoria académica. En nuestro caso pensamos que tal circunstancia se puede atajar, si el alumnado ha cursado previamente la asignatura sobre redacción de textos legales, ofertada en los estudios ordinarios del Grado en Derecho.

Finalizamos este apartado con algunos comentarios acerca de la formación a distancia, que podría implementarse en un entorno virtual provisto de herramientas tales como un correo y foros de debate. En la época actual, donde las nuevas tecnologías van ganando terreno paulatinamente a las sesiones presenciales, esta opción resulta sugerente por varias razones. Entre ellas, la de que se trata de un cauce muy interesante para acercar la formación al alumnado que no puede asistir a clase por diferentes motivos (incompatibilidad de horarios, desplazamientos costosos, etc.). Sin embargo, esta modalidad y otras semipresenciales (si bien en este caso en menor medida) suscitan varios aspectos para la reflexión que aunque no cuestionan una posible implementación, sí plantean cuestiones a tener en cuenta o posibles limitaciones. Tenemos experiencia en docencia presencial pero no a distancia. No obstante, venimos considerando esta última

\footnotetext{
${ }^{114}$ BERGERON, R. C. "The Training of Drafters", cit., p. 105.

${ }^{115}$ WEBSTER, R. "Teaching legislative drafting...", cit., p. 53.

${ }^{116}$ GWIZDZ, A. "Organization of the Post-Graduate Course...”, cit., p. 109. La última opción señalada la ponemos en práctica en UNIVERSIDAD DE ALCALÁ. Redacción de Textos Legales, cit., p. 7.

${ }^{117}$ CENTENERA SÁNCHEZ-SECO, F. "Recursos para formar...”, cit., p. 147.
} 
a modo de proyecto. En base a ello podemos dejar los siguientes comentarios. En primer lugar, pensamos que en la formación a distancia el desarrollo de explicaciones o aclaraciones de dudas seguramente resulte más complejo que en el caso del formato presencial. Es probable que varios de los temas que se aborden susciten preguntas entre el alumnado; incluso las respuestas den posiblemente paso a otros interrogantes. La gestión de esta circunstancia entendemos que puede desarrollarse con mucha más fluidez en el aula que en un entorno virtual. Conviene además señalar que en el ámbito presencial es posible llevar a cabo dinámicas de trabajo, cuya implementación parece más compleja en un entorno virtual. Pensamos, por ejemplo, en el modelo que sugiere el trabajo del alumnado en pequeños grupos, con el fin de resolver los ejercicios propuestos. Quisiéramos señalar también que nuestra experiencia docente nos demuestra que en las sesiones presenciales, tanto teóricas como prácticas, las participaciones del alumnado enriquecen los contenidos y las posibilidades de resolución de ejercicios; algo que, a nuestro modo de ver, no parece que pueda darse, al menos en la misma medida, en el modelo a distancia. Finalmente, debe tenerse en cuenta que esta última opción conlleva una carga de trabajo considerable para el profesorado, no únicamente en lo que respecta al diseño del entorno docente, sino principalmente a propósito de las labores que tienen que ver, por ejemplo, con la retroalimentación individual que debería facilitarse por cada ejercicio realizado ${ }^{118}$. En la modalidad presencial es suficiente con corregir en una ocasión los ejercicios desarrollados en el aula (salvo en el caso de que se desarrollen pruebas individuales escritas, que sí requieren una retroalimentación individual), atendiendo a las posibles dudas o comentarios que pudieran surgir con ocasión de la corrección.

\section{CONCLUSIONES}

Desde hace años, numerosos estudios vienen señalando que en la formación jurídica se está olvidando la actividad creadora del derecho. Para hacer referencia a aquella en este trabajo hemos utilizado el término legística, que comprende aspectos formales como la redacción (aunque en conexión con otros niveles de racionalidad), pero también otros materiales que tienen que ver con la determinación de contenidos. En nuestros días se pueden observar avances y experiencias en numerosos países, pero en otros apenas se perciben. Este estudio pretende reivindicar la importancia de la formación en cuestión. Para ello, aporta respuestas a los siguientes interrogantes.

¿Por qué formar en legística? En primer lugar, porque una formación restringida a los ámbitos de la interpretación y aplicación del derecho es incompleta y carece de la calidad deseada. En segundo lugar, porque el alumnado que estudia derecho probablemente tenga que redactar textos legales en su futura vida laboral. La ausencia de formación en este ámbito se traducirá en falta de competencia y ello, a su vez, en la contravención de deberes deontológicos que solicitan profesionales con buena formación. En el contenido de esta pueden observarse variaciones, en función del modelo de enseñanza que se desarrolle. En base a la experiencia docente que tenemos sobre la materia, podemos decir que la formación en cuestión alcanza a los aspectos lingüísticos, los que tienen que ver con otros niveles de racionalidad de la legística y los relativos a aquellos conocimientos jurídicos necesarios para desarrollar los ejercicios prácticos propuestos. La tercera razón que puede aportarse en este punto es que la

\footnotetext{
${ }^{118}$ Desarrollamos esta cuestión teniendo en cuenta lo que señala CRABBE, V. “The ethics...”, cit., p. 12.
} 
formación que nos ocupa supone apostar por determinados postulados del Estado de derecho y por un resultado legislativo de calidad, capaz de superar numerosos test de racionalidad.

¿Dónde y cuándo formar? Probablemente la opción más extendida sea aquella que nos invita a fijar la mirada en los estudios de postgrado, llevados a cabo tanto por instituciones gubernamentales, como por universidades o por la conjunción de ambas. Sin embargo, también son numerosas las experiencias que se están implementando en los estudios ordinarios. El desarrollo en este ámbito pensamos que resulta especialmente sugerente, además de porque supone una aportación muy considerable a la consecución de los objetivos citados en el párrafo precedente, porque quizá sea el único cauce para hacer llegar la formación a determinadas trayectorias académicas (no en todo caso se hace un postgrado). En esta parcela puede pensarse en un programa desarrollado en asignaturas ofertadas a lo largo de la carrera. Conviene, no obstante, apuntar que también puede resultar interesante dedicar la temática del trabajo de fin de grado a la legística. Además, cabe añadir que la celebración de eventos ofrece numerosas oportunidades para la formación. Por otra parte, aquella también podría desarrollarse en base a un modelo de enseñanza a distancia.

¿En qué formar? En algunos estudios se considera que a la hora de plantear la formación que nos interesa han de tenerse en cuenta materias como derecho constitucional o administrativo. En ello, no obstante, no existe consenso. A nuestro modo de ver no es necesario transmitir muchos conocimientos sobre una o varias materias jurídicas, sino facilitar aquellos que incidan directamente en la labor a realizar. En todo caso, lo cierto es que un programa sobre legística requiere centrar la atención en disciplinas específicas, que no forman parte del currículum tradicional. En ocasiones se ha propuesto el siguiente índice temático: teoría de la legislación, analítica de la legislación, táctica de la legislación, metódica de la legislación y técnica de la legislación. La legística, según la hemos entendido en este estudio, comprendería las dos últimas materias de la relación y parte de la penúltima. Los contenidos de las experiencias llevadas a cabo pueden enmarcarse en esta acotación temática -aunque en ocasiones se incluyen otras disciplinas de la relación, o bien algunas que pueden contextualizarse en la reflexión inicial de esta conclusión-, si bien es cierto que los programas suelen centrar la atención en algunos de los ámbitos señalados. Debe apuntarse además que determinadas circunstancias y particularidades, propias del contexto en el que se ha implementado la formación, han contribuido a matizar o desarrollar en un cierto sentido el esquema expuesto.

¿Cómo formar? En la formación deben transmitirse contenidos, pero también es necesario entrenar en habilidades a propósito de diferentes ámbitos. En relación a este último aspecto, debe tenerse en cuenta que las labores de legística, tanto en su perspectiva formal como material, no suponen exclusivamente prácticas desarrolladas en base a pautas científicas. En aquellas existe también un componente importante de arte que conlleva práctica y especulación, algo que hace ineludible el entrenamiento en habilidades. Conviene apuntar además que el ámbito en el que se desarrolla la creación del derecho tiene numerosas características, que lo diferencian de aquel en el que se aplica e interpreta (así, por ejemplo, en lo que respecta al margen de decisión, el planteamiento del razonamiento básico, el objeto de análisis o la tipología de casos que se tratan). Dichas particularidades han de tenerse en cuenta a la hora de plantear la formación en habilidades y las prácticas en el marco docente que nos interesa. 
Para llevar a cabo los objetivos señalados en el párrafo anterior se pueden desarrollar varias metodologías. Los contenidos pueden transmitirse mediante clases magistrales, pero también con otras dinámicas recomendables que propician la participación del alumnado. Por otra parte, en relación a la adquisición de habilidades existen numerosas propuestas prácticas de complejidad variable para formar al alumnado, tanto en lo que respecta a la redacción propiamente dicha, como en lo que se refiere a la gestión de aspectos tales como el establecimiento de objetivos o las medidas para que puedan conseguirse aquellos. A nuestro modo de ver, la formación ideal en legística sería aquella que combinase ambos ámbitos, algo que podría ser especialmente factible -principalmente porque se trata de las prácticas que ofrecen una cobertura temporal más amplia- en el caso de los trabajos de fin de curso o de grado. La implementación del modelo a distancia puede plantearse a partir de un entorno docente virtual. No obstante, en comparación con el presencial aquel parece presentar mayores inconvenientes en aspectos tales como la explicación de contenidos, la resolución de dudas, las dinámicas de trabajo que puede desarrollar el alumnado, o la carga de trabajo para el profesorado.

\section{BIBLIOGRAFÍA}

AA.VV. A feitura das leis, tomo I, Oeiras, 1986.

AA.VV. A feitura das leis, tomo II, Oeiras, 1986.

AA.VV. Curso de técnica legislativa GRETEL, Madrid, 1989.

AA.VV. III Jornadas de Derecho Parlamentario. La función legislativa de los parlamentos y la técnica de legislar, Madrid, 2000.

AA.VV. Legislador i tècnica legislativa. Workshop celebrat al Palau del Parlament el dia 17 de Febrer de 2003, Barcelona, 2003.

ATIENZA, M. Contribución a una teoría de la legislación, Madrid, 1997.

BENTHAM, J. Nomografía o el arte de redactar leyes, Madrid, 2004.

BERGEL, J.-L. "The Drafting of the Norm", AA.VV. (Karpen, U. y Delnoy, P., ed.) Contributions to the Methodology of the Creation of Written Law, Baden-Baden, 1996.

BERGERON, R. C. “The Training of Drafters", AA.VV (Karpen, U. y Delnoy, P., ed.) Contributions to the Methodology of the Creation of Written Law, Baden-Baden, 1996.

BLANCO DE MORAIS, C. Manual de Legística. Criterios Científicos e Técnicos para Legislar Melhor, [s. 1.], 2007.

BOBBIO, Teoría General del Derecho, Bogotá, 2005.

CARRILlO GARCÍA, C. Y. "Calidad de las leyes. Algunos puntos críticos", Legislação. Cadernos de Ciencia de Legislação, 52, 2010, pp. 5-41.

CAUPERS, J. "Relatório sobre o programa, conteúdo e métodos de uma disciplina de Metódica da Legislação", Legislação. Cadernos de Ciencia de Legislação, 35, 2003, pp. 5-87.

CENTENERA SÁNCHEZ-SECO, F. "Buscando el valor de la claridad de las normas: algunas reflexiones desde el pensamiento de Lon L. Fuller", Bajo Palabra. Revista de Filosofía, 10, 2015, pp. 61-70. 
CENTENERA SÁNCHEZ-SECO, F. "La claridad legislativa en el pensamiento de Lon L. Fuller: un análisis desde la teoría de la legislación”, Anales de Derecho, 1, 2015, pp. $1-27$.

CENTENERA SÁNCHEZ-SECO, F. "La crisis de la ley en Luigi Ferrajoli: algunas consideraciones desde la teoría de la legislación", Cuadernos Electrónicos de Filosofía del Derecho, 26, 2012, pp. 283-309.

CENTENERA SÁNCHEZ-SECO, F. "Los criterios lingüísticos de las directrices de técnica normativa: una propuesta para la solución de enfrentamientos entre principios", Revista de Llengua i Dret, 58, 2012, pp. 15-34.

CENTENERA SÁNCHEZ-SECO, F. "Recursos para formar en redacción de textos legales", Revista de Llengua i Dret, 65, 2016, pp. 136-148.

COMISIÓN DE EXPERTOS MODERNIZACIÓN DEL LENGUAJE JURÍDICO. Informe de la Comisión de modernización del lenguaje jurídico, [s. 1.], 2001. Disponible en: $\quad \mathrm{http}: / /$ lenguajeadministrativo.com/wp-content/uploads/2013/05/cmljrecomendaciones.pdf. Fecha de consulta: 24/04/2016.

CRABBE, V. "The ethics of legislative drafting", Commonwealth Law Bulletin, 36, 1, 2010, pp. 11-24.

DE MONTALIVET, P. "La «juridicisation» de la légistique. À propos de l'objectif de valeur constitutionnelle d'accessibilité et d'intelligibilité de la loi", AA.VV. (Drago, R., dir.) La confection de la loi, Paris, 2005.

DICK, R. C. Legal Drafting in Plain Language, Ontario, 1995.

DRINÓCZI, T. "Quality Drafting-The case of Hungary", Legisprudence. International Journal for the Study of Legislation, IV, 2, 2010, pp. 157-170.

DUPRAT, J.-P. "The French »learning" legislator: the improvements of an indirect proces", AA.VV. (Mader, L. y Moll, C., ed.) The Learning Legislator, Baden-Baden, 2009.

FULLER, L. L. La moral del derecho, México, 1967.

FULLER, L. L. "What the Law Schools Can Contribute to the Making of Lawyers", Journal of Legal Education, 1, 2, 1948, pp. 189-204.

GALIANA SAURA, A. La legislación en el Estado de Derecho, Madrid, 2003.

GALIANA SAURA, A. La ley: entre la razón y la experimentación, Valencia, 2008.

GARCÍA-ESCUDERO MÁRQUEZ, P. Técnica legislativa y seguridad jurídica: ¿hacia el control constitucional de la calidad de las leyes?, Navarra, 2010.

GOMES CANOTILHO, J. J. "Relatório sobre programa, conteúdos e métodos de um curso de teoria da legislação", Boletim da Faculdade de Direito, LXIII, 1987, pp. 405494.

GWIZDZ, A. "Organization of the Post-Graduate Course in Legislation at the University of Warsaw", AA.VV. (Karpen, U. y Delnoy, P., ed.) Contributions to the Methodology of the Creation of Written Law, Baden-Baden, 1996.

ILES, W. "Coment", AA.VV. Law Commission. Legislation and its interpretation. Discussion and seminar papers, New Zealand, 1989. 
KARPEN, U. "Improving Democratic Development by Better Regulation", AA.VV. (Stefanou, C. y Xanthaki, H., ed.) Drafting Legislation. A Modern Approach, England, 2008.

KARPEN, U. "Law Drafting and the Legislative Process: outline of a training course for law drafters", AA.VV. (Mader, L. y Moll, C., ed.) The Learning Legislator, BadenBaden, 2009.

KARPEN, U. "Resumé”, AA.VV. (Karpen, U., ed.) Legislation in European Countries, Baden-Baden, 1996.

KELLY, D. "The Victorian Experience of Plain Drafting”, AA.VV. Law Commission. Legislation and its interpretation. Discussion and seminar papers, New Zealand, 1989.

KRONGOLD, S. "Writing Laws: making them easier to understand", Ottawa Law Review, 24, 2, 1992, pp. 495-581.

MADER, L. "Legislative Procedure and the Quality of Legislation", AA.VV. (Karpen, U. y Delnoy, P., ed.) Contributions to the Methodology of the Creation of Written Law, Baden-Baden, 1996.

MADER, L. "Legistic Training and Education in Switzerland", AA.VV. (Mader, L. y Moll, C., ed.) The Learning Legislator, Baden-Baden, 2009.

MARCELLO, D. A. "Legislative Learning in the United States and Europe: How jurisdictions differ in training drafters and enacting laws", AA.VV. (Mader, L. y Moll, C., ed.) The Learning Legislator, Baden-Baden, 2009.

MARCILLA CÓRDOBA, G. “Argumentación jurídica y racionalidad legislativa en el Estado Constitucional”, Anuario de Filosofía del Derecho, 21, 2004, pp. 337-351.

MARCILLA CÓRDOBA, G. “Argumentación legislativa y teoría estándar de la argumentación jurídica", AA.VV. (Gascón Abellán, M., coord.) Argumentación jurídica, Valencia, 2014.

MARCILLA CÓRDOBA, G. "Justificación de las decisiones legislativas: un corolario del estado constitucional”, AA.VV. (Gascón Abellán, M., coord.) Argumentación jurídica, Valencia, 2014.

MARCILLA CÓRDOBA, G. Racionalidad legislativa. Crisis de la ley y nueva ciencia de la legislación, Madrid, 2005.

MARCILLA CÓRDOBA, G. "Sobre la necesidad de una nueva ciencia de la legislación", AA.VV. (Carbonell, M., Thalía, S., coord.) Elementos de técnica legislativa, México, 2000.

MARKMAN, S. C. "Training of Legislative Counsel: learning to draft without Nellie", Commonwealth Law Bulletin, 36, 1, 2010, pp. 25-39.

MARTINO, A. A. Manual de Técnica Legislativa. Disponible en: http://www.antonioanselmomartino.it/Digesto/manual.html. Fecha de consulta: $10 / 05 / 2016$.

MORAN, E. "Foreword”, Commonwealth Law Bulletin, 36, 1, 2010, pp. 1-6.

MORAND, C.-A. "Éléments de légistique formelle et matérielle", AA.VV. (Morand, C.-A., dir.) Légistique formelle et matérielle, Aix-en-Provence, 1999.

MORAND, C.-A. "Préface", AA.VV. (Morand, C.-A., dir.) Légistique formelle et matérielle, Aix-en-Provence, 1999. 
MURO RUIZ, E. "Enseñanza de la técnica legislativa", Academia. Revista sobre enseñanza del Derecho, 11, 2008, pp. 63-91.

NAMPOOTHIRY, N. K. "The role of Parliamentary Counsel in legislative drafting", Commonwealth Law Bulletin, 36, 1, 2010, pp. 57-65.

PAGANO, R. Introduzione alla legistica. L'arte di preparare le leggi, Milano, 2001.

PINEDA GARFIAS, R. "Teoría de la Legislación. Algunos planteamientos generales", Revista de Derechos Fundamentales, 3, 2009, pp. 137-156.

POPELIER P. y VELINDER, V. "Framework for a Country Comparative perspective: Belgium”, AA.VV. (Mader, L. y Moll, C., ed.) The Learning Legislator, Baden-Baden, 2009.

PRIETO DE PEDRO, J. "Lenguaje jurídico y Estado de Derecho", Revista de Administración Pública, 140, 1996, pp. 111-129.

SALVADOR CODERCH, P. "Elementos para la definición de un programa de técnica legislativa”, AA.VV. (Cavero Gómez, M., coord.) La función legislativa de los parlamentos y la técnica de legislar, Madrid, 2000.

SALVADOR CODERCH, P. “Técnica legislativa y teorías de la regulación”, InDret, 2, 2004, pp. 1-28.

SEMENOVA, N. "Teaching the Learning Legislator in Ukraine: A country presentation", AA.VV. (Mader, L. y Moll, C., ed.) The Learning Legislator, BadenBaden, 2009.

STARK, J. The Art of the Statute, Littleton, 1996.

SVENSSON, P. A. "The Swedish way to create lawful, consistent and uniform legislation - a summary presentation", AA.VV. (Mader, L. y Moll, C., ed.) The Learning Legislator, Baden-Baden, 2009.

TAMANAHA, B. Z. "A Concise Guide to the rule of law", AA.VV. (Mader, L. y Moll, C., ed.) The Learning Legislator, Baden-Baden, 2009.

TAVARES DE ALMEIDA, M. "Country presentations: Portugal”, AA.VV. (Mader, L. y Moll, C., ed.) The Learning Legislator, Baden-Baden, 2009.

TAVARES DE ALMEIDA, M. "Legista - uma nova profissão”, Legislação. Cadernos de Ciencia de Legislação, 41, 2005, pp. 35-43.

THE OFFICE OF LEGISLATIVE LEGAL SERVICES. Colorado Legislative Drafting Manual. Disponible en: http://tornado.state.co.us/gov_dir/leg_dir/olls/LDM/OLLS_Drafting_Manual.pdf. Fecha de consulta: 10/05/2016.

THORNTON, G. C. Thornton's Legislative Drafting, [London], 2013.

UNIVERSIDAD DE ALCALÁ. Redacción de Textos Legales. Disponible en: http://www.uah.es/estudios/asignaturas/programas/G61/100076_G61_2015-16.pdf.

Fecha de consulta: 30/04/2016.

UNIVERSITÀ DI PISA. Master in Scienza della legislazione. Disponible en: http://www.altalex.com/index.php?idnot=3833. Fecha de consulta: 24/04/2016.

UNIVERSITÉ DE GENÈVE. Rédaction juridique. Disponible en: http://wadme.unige.ch:3149/pls/opprg/w_det_cours.debut?p_code_cours=5330\&p_plan 
is $=0 \& p \_$langue $=1 \& p \_$frame $=N \& p \_$mode $=P G C \& p \_$annee $=2014 \& p \_s u f f i x e=\& p \_g r t r i$ $=12490$. Fecha de consulta : 24/04/2016.

UNIVERSITY OF LONDON. Sir William Dale Legislative Drafting Clinic. Disponible en: http://ials.sas.ac.uk/postgrad/LDclinic/LDclinic.htm. Fecha de consulta: 24/04/2016.

VAN LOCHEM, P. J. P. M. "The Dutch Academy for Legislation", AA.VV. (Mader, L. y Moll, C., ed.) The Learning Legislator, Baden-Baden, 2009.

WEBSTER, R. "Teaching legislative drafting: reflections on the Commonwealth Secretariat Short Course", Commonwealth Law Bulletin, 36, 1, 2010, pp. 41-56.

WRONKOWSKA S. y GWIZDZ, A. "Poland”, AA.VV. (Karpen, U., ed.) Legislation in European Countries, Baden-Baden, 1996.

XANTHAKI, H. Drafting Legislation. Art and Technology of Rules for Regulation, Oxford, 2014.

ZAPATERO, V. "De la jurisprudencia a la legislación”, Doxa, 15-16, 1994, pp. 769789.

ZAPATERO, V. El Arte de Legislar, Pamplona, 2009.

ZAPATERO. V. "El arte ilustrado de legislar", Bentham, J. Nomografía o el arte de redactar leyes, Madrid, 2004. 